\title{
1 Generative models, language and active inference
}

${ }^{a}$ The Wellcome Centre for Human Neuroimaging, UCL Queen Square Institute of Neurology, 12 Queen Square, London, WC1N 3AR, U.K.

${ }^{b}$ Virtual Structures Research, Inc., 12204 Saint James Rd, Potomac, MD 20854, U.S.A.

Emails: k.friston@ucl.ac.uk, thomas.parr.12@ucl.ac.uk, imc.yufik@att.net, noor.sajid.18@ucl.ac.uk,

Declarations of interest: none

* Correspondence: Emma Holmes; The Wellcome Centre for Human Neuroimaging; UCL Queen emma.holmes@ucl.ac.uk 


\section{Abstract}

24 This paper presents a biologically plausible generative model and inference scheme that is capable of simulating the generation and comprehension of language, when synthetic subjects talk to each other.

26 Building on active inference formulations of dyadic interactions, we simulate linguistic exchange to 27 explore generative models that support dialogues. These models employ high-order interactions among abstract (discrete) states in deep (hierarchical) models. The sequential nature of language processing mandates generative models with a particular factorial structure - necessary to accommodate the rich combinatorics of language. We illustrate this by simulating a synthetic subject who can play the 'Twenty Questions' game. In this game, synthetic subjects take the role of the questioner or answerer, using the same generative model. This simulation setup is used to illustrate some key architectural points and demonstrate that many behavioural and neurophysiological correlates of language processing emerge under variational (marginal) message passing, given the right kind of generative model. For example, we show that theta-gamma coupling is an emergent property of belief updating, when listening to another.

Keywords: language; hierarchical; inference; Bayesian; neuronal; connectivity; free energy; message passing 


\section{1. Introduction}

43 In April, 2018, an international group of experts assembled in Frankfurt for an Ernst Strüngmann forum addressing complexity and computation in the cortex (Singer et al., 2019). One group was briefed to discuss human cognition and, reflecting the interests of that group, chose to focus on language processing - a challenging area for computational neuroscience, linguistics and theoretical neurobiology (Hauser et al., 2002). What follows is a formal analysis that speaks to a key conclusion of that group; namely, that the neuronal correlates of language processing and functional brain architectures should emerge naturally, given the right kind of generative model. In brief, this paper uses simulations of language processing to show that many behavioural and neurophysiological correlates of language processing emerge under deep diachronic models.

A generative model refers to a probabilistic mapping from causes (e.g., semantics) to consequences (e.g., auditory signal). Perception, recognition or inference then becomes the (Bayesian) inversion of the generative model to infer causes from consequences. The notion of a generative model rests on a commitment to the brain as a constructive organ, generating explanations for its sensations. We will use an active inference (a.k.a., predictive processing) formulation of this basic idea that inherits from a long tradition of psychological ideas about how the brain works; from Kant through Helmholtz (Helmholtz, 1878 (1971)), from analysis by synthesis (Yuille and Kersten, 2006) to perception as hypothesis testing (Gregory, 1980), from the Helmholtz machine (Dayan et al., 1995) to the free energy principle (Friston, 2010). Specifically, we will use a corollary of the free energy principle; namely, active inference (Friston et al., 2017a). The basic idea behind active inference is that any neuronal processing can be formulated, in a normative sense, as a minimisation of the same quantity used in approximate Bayesian inference; i.e., a variational free energy or evidence bound (Mattys et al., 2005; Winn and Bishop, 2005).

Minimizing variational free energy is equivalent to maximizing the sensory evidence for an internal model of how unobserved (i.e., hidden) states of the world generate observed (i.e., sensory) 
Modelling 'Twenty Questions'

consequences. Technically, this can be formulated in terms of maximising the marginal likelihood for models of the lived world - that is neatly summarized as self-evidencing (Clark, 2016; Hohwy, 2016); in other words, gathering sensory evidence for our generative models. Having specified the generative model one can then use standard, 'off-the-shelf' belief updating schemes (Friston et al., 2017c) to create synthetic agents, who perceive and act in a self-evidencing fashion. These simulations can also be used to predict empirical behavioural and physiological responses. Here, we use simulations to test hypotheses about language; such as generation and understanding, in relation to conceptual knowledge (Barsalou, 2003; Yufik, 1998; Yufik, 2019), the use of a shared narrative (Mar, 2011; Mathewson et al., 2019), and the linearization of language (Bornkessel et al., 2005).

This paper extends a long line of existing work in the domain of natural language processing (and response generation). Previously, the focus has been on treating natural language processing as a learning problem (Elman, 1990), where the use of deep learning has spearheaded algorithmic developments (Young et al., 2018): e.g., word embeddings derived from learning predictive relationships (Collobert et al., 2011; Mikolov, 2010; Mikolov et al., 2013; Pennington et al., 2014) and fully contextualized word representations (Devlin et al., 2018; Radford et al., 2019; Vaswani et al., 2017). These approaches to natural language processing limit themselves to learning associationsbetween an input and output-via the training of particular neural networks. In contrast, response generation —including conversational dialogue agents—-have been framed as either deep reinforcement learning (Li et al., 2016; Zhao et al., 2017) or inference problems (Liu et al., 2018). These approaches, whilst closely aligned with our work, are optimising objective functions that do not account for the future, including their ability to have forward-looking conversations, due to word-repetitions or closedform replies (Li et al., 2016). In contrast, by framing language as an active (Bayesian) inference problem, with an underlying generative model, our approach infers causal relationships between inputs and outputs - and provides a structural understanding of the sequences of words being presented and their context sensitivity. This results in uncertainty resolving actions that lead to forward-looking 
Modelling 'Twenty Questions'

92

conversations: as demonstrated in the simulations that follow, an agent does not need to revisit issues that have already been resolved.

The resulting approach also differs from previous cognitive theories of language processing. Although the idea of 'surprisal' has become increasingly prevalent in the literature (Hale, 2001; Levy, 2008), this usually refers to the magnitude of 'surprise' conveyed by individual words, such that expected semantics are simply an amalgamation of the semantics conveyed by all preceding words. In contrast, in the current formulation, belief updating occurs at a higher level and relies on beliefs about an acoustic scene, about which the agent has prior beliefs. Note that the mathematical formulation used herewhich is described in detail in the sections that follow-differs from previous approaches in this literature. There are two key points to note here. First, the current formulation considers the uncertainty of the agent's beliefs about the scene at hand. Second, we introduce an active component-which generates predictions about the information that an agent will seek to resolve their uncertainty. In other words: What questions should I ask next, to resolve my uncertainty about the subject of our conversation?

This paper comprises four sections. The first (Generative models of language) describes a top-down approach to understanding functional brain architectures in terms of generative models, with a special focus on models that are apt for language processing. This section considers the requisite computational architecture and the second section (Active inference) describes the accompanying message passing. The third section ("Twenty Questions" simulations) uses the generative model to illustrate behavioural and neurophysiological correlates of speaking and listening (Edwards and Chang, 2013; Kayser, 2019; Lizarazu et al., 2019; Pefkou et al., 2017). This section concludes with a reproduction of some simple (violation) paradigms, in terms of synthetic event related potentials and difference waveforms - of the sort seen in mismatch negativity, P300, and N400 studies (Coulson et al., 1998; Van Petten and Luka, 2012). The final section (Synthetic communication) turns to communication per se, using dyadic interactions between two synthetic subjects to illustrate that certain kinds of belief updating can be 
Modelling 'Twenty Questions'

117 instantiated linguistically. We conclude with a discussion of what has not been addressed; for example, 118 a sense of agency and the acquisition of language through learning deep models.

\section{Generative models of language}

121 The advantage of focusing on generative models for language - as opposed to recognition models - is that the same generative model can be used to generate an auditory signal given a narrative (i.e., for language production) and to infer the narrative given auditory input (i.e., for language understanding). Here, we focus on simulating a simple agent, who can ask questions and answer them. In this formulation, the agent does not know whether its beliefs are its own or are generated by some external narrator. We will return to this issue in the discussion.

So, what are the special requirements of a generative model for language? Here, we take a commonsense approach and list the necessary properties such a model must possess. Starting with the generative model somewhat simplifies things, in the sense that one only has to specify what would be sufficient to generate meaningful language. One can then use established inversion schemes for language understanding. First, we will assume that language is for communication, which immediately implies a shared forward-looking narrative (Allwood et al., 1992; Brown and Brune, 2012; Friston and Frith, 2015a; Mar, 2011; Schegloff and Sacks, 1973; Specht, 2014). In turn, this implies shared (and evolving) beliefs about the subject of communication (Mathewson et al., 2019). This simple observation has some fundamental implications. The first may be slightly counterintuitive and borrows from earlier work on neuronal hermeneutics (Friston and Frith, 2015a). This work — using generalised synchrony to simulate communication between songbirds - suggests that it is sufficient to share the same generative model to infer the meaning of sensory exchanges between interlocutors. The issue of who is talking and attribution of agency then becomes a somewhat secondary issue, which is only necessary for turn-taking

140 (Ghazanfar and Takahashi, 2014; Wilson and Wilson, 2005). In short, a narrative cannot be uniquely attributed to you or me-it is our narrative. 
Modelling 'Twenty Questions'

142 The notion of a shared narrative is central to our formulation of language. Usually, in realising or 143 simulating active inference (in real artefacts or in silico), outcomes are generated by external states of 144 the world that agents navigate. These sensory outcomes are then used to update beliefs about external states, which are used to plan actions. Policies — which are sequences of actions — change external states and generate new outcomes. And so, the perception-action cycle continues. However, in the context of dyadic exchange, outcomes are generated by another person or agent, without any necessary reference to external states. In this setting, when an artificial agent speaks, it generates outcomes that are most consistent with its beliefs which, in turn, update the beliefs of its correspondent. The upshot of this exchange is a synchronisation or alignment of belief states that —in pure communication—circumvent any reference to external states of the world.

This alignment follows naturally from generating outcomes that are consistent with beliefs (technically, outcomes that have the greatest marginal likelihood or model evidence). Actions and outcomes are assumed to be isomorphic. Subsequent belief updating based on those outcomes makes the beliefs of both subjects consistent with the outcomes they share. In short, outcomes and beliefs are selected in concert to maximise model evidence and, implicitly, the predictability of sensory samples. The inevitable endpoint of this reciprocal exchange is convergence to the same belief states (Isomura et al., 2019), which ensure the outcomes are generated by one agent are easily predictable, in virtue of the fact that these are the same outcomes the agent would have produced itself. This kind of generalised synchronisation has been explored in numerical analyses of communication by birdsong and intracellular communication (Friston et al., 2015; Friston and Frith, 2015b; Isomura et al., 2019; Kuchling et al., 2019). In this paper, we focus on pure communication; in the sense that all outcomes are generated by one or another agent. This means there are no other states of the world to consider.

164 See Figure 1 for a graphical depiction of the special conditional dependencies implied by pure 165 communication. 

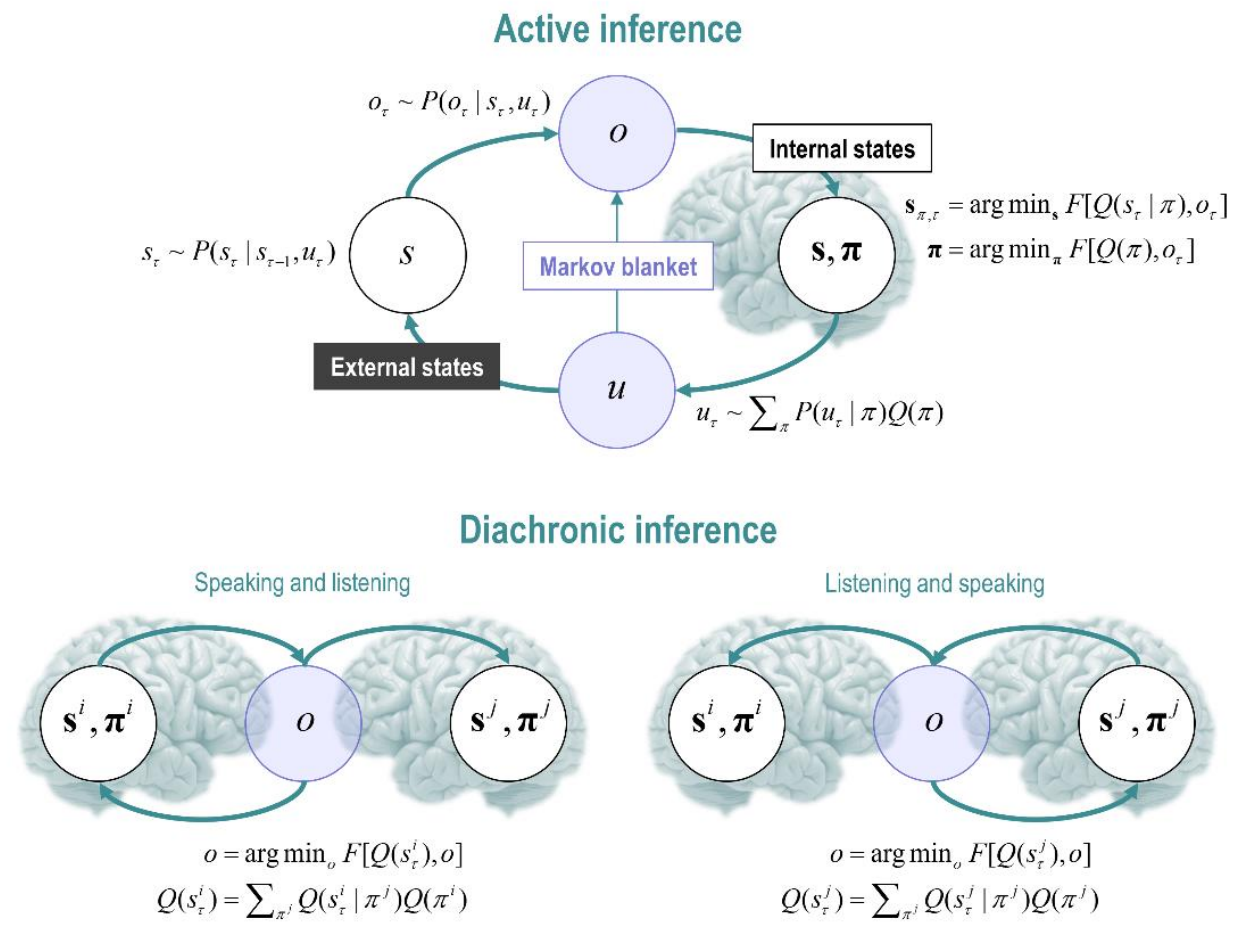

Active inference and Markov blankets. This figure illustrates the conditional dependencies among various states that constitute (active) inference about external states of affairs in the world. Active inference rests upon a fourway partition of states into external states $(s)$ and internal states $(\mathbf{s}, \boldsymbol{\pi})$ that are separated by Markov blanket states $(o, u)$. Technically, the Markov blanket of internal states comprises their parents, their children and the parents of the children. In this figure, blanket states correspond to the pale blue circles. Blanket states comprise observations or outcomes $(o)$ and action $(u)$. The upper panel illustrates the standard way in which conditional dependencies are mediated: internal states are treated as encoding representations of external states. These representations prescribe action on external states, which generates outcomes. In this construction, internal states play the role of sufficient statistics or parameters of a posterior belief $(Q)$ about external states and plans or policies that are realised by action. These beliefs are optimised by minimising a free energy functional of posterior beliefs, given outcomes. Posterior beliefs about the policies provide a probability distribution from which the next action is sampled. This action changes external states, which generate outcomes - and so the (perception-action) cycle continues. The lower panel shows the simplified scheme used in this paper, labelled 'Diachronic inference'. In this setting, actions $(u)$ and outcomes $(o)$ are assumed to be isomorphic. In other words, I act by generating an outcome that minimises free energy. This is equivalent to generating or selecting outcomes that are the most likely under my beliefs about the causes of that outcome. Because these outcomes are shared between two (or more) agents, they constitute the Markov blanket that separates the internal states of every agent in the exchange. This means the internal states of one agent now constitute the external states of another (and vice versa). Crucially, this rests upon a diachronic switching, in which only one agent generates outcomes at any one time. Heuristically, this 
Modelling 'Twenty Questions'

means that I can either listen or speak but not both at once. With this particular constraint on conditional dependencies, the shared outcome is (e.g., spoken words) constitute the blanket states that are shared by all agents. The superscripts in the lower panel denote two agents $(i$ and $j$ ). The equations express the sampling of various states, or their minimisation with respect to variational free energy. An interesting aspect of the diachronic setup is that everything minimises a free energy; effectively resolving uncertainty; such that the beliefs of one agent are installed in another, via an exchange of outcomes.

In what follows, we try to show how the belief states of two or more agents become aligned through pure communication, where this alignment is an emergent property of selecting beliefs that are consistent with what is heard while, at the same time, generating outputs that are consistent with those beliefs. If two or more agents comply with these imperatives, their beliefs align, thereby evincing a minimal form of communication. It is interesting to consider how external states might get into the game; for example, providing visual cues that affect the beliefs of one agent: i.e., how does one person convey her beliefs about what she is seeing to another, or how do they reach consensus when they can see different parts of the same scene? However, in this work, we will just consider pure communication without external states and focus on how beliefs about a scene are installed by a shared narrative.

So, what is a narrative? On the active inference view, everything we do can be regarded as pursuing a narrative that resolves uncertainty (Friston et al., 2017a; Mirza et al., 2016). This means that the only sort of narrative that matters is one that has epistemic affordance; namely, the opportunity to reduce uncertainty under a particular belief structure about the world. In this sense, the formal imperatives for language become exactly the same as any active inference; for example, active vision (Ferro et al., 2010; Ognibene and Baldassarre, 2014). Indeed, the same principles underlie experimental design in scientific enquiry, where one solicits data that disambiguate among competing hypotheses (Lindley, 1956). Much of the motivation for the generative model below inherits from the formally identical problem of querying the world with saccadic eye movements during scene construction (Mirza et al., 2016; Ognibene and Baldassarre, 2014; Purpura et al., 2003). In short, we will regard language as acting on 
Modelling 'Twenty Questions'

214 the world to resolve uncertainty, where epistemic foraging has been elevated from visual palpation (i.e.,

215 visual search in active vision) to an interrogation of the world via semantics and semiotics, acquired by

216 encultured learning (Constant et al., 2019; Creanza and Feldman, 2014; Penn et al., 2008; Rizzolatti

217 and Craighero, 2004). Following this analogy to its conclusion, a minimal but sufficient model of

218 language can then be framed in terms of a series of 'questions' and 'answers' (i.e., propositions and

219 responses), in the same way that sampling the world with our sensory epithelia constitutes a 'question

220 about what is out there' (Gregory, 1980). And the subsequent sensory samples provide some salient,

221 uncertainty reducing, evidence for our beliefs about the world.

222 With this in mind, we set ourselves the task of formulating a generative model that could play a game of "Twenty Questions". In other words, a model that could generate a sequence of questions and closed "yes/no" answers, which progressively reduce uncertainty about the subject of conversation (i.e., contextual knowledge). These sorts of sequential language games have extensively been tackled in the literature: including one round of question-answer 'whisky pricing' interaction (Hawkins et al., 2015), playing restricted 'cards corpus' with one-off communication (Potts, 2012), sequential 'info-jigsaw' game (Khani et al., 2018), 'hat game' where agents learn to communicate via observing actions (Foerster et al., 2016) and conversations about visual stimulus (Das et al., 2017). Having specified the generative model for our "Twenty Questions" paradigm, we made no further assumptions — we used off-the-shelf (marginal) message passing to simulate neuronal processing (Dauwels, 2007; Friston et al., 2017c; Parr and Friston, 2018; Winn and Bishop, 2005). Exactly the same belief updating scheme, for partially observed Markov decision processes, has been used in many contexts; ranging from exploration of mazes and economic game theory, through to abstract rule solving and scene construction (Friston et al., 2017a). We anticipated that these simulations would reproduce key behavioural and neuronal responses seen in empirical language studies. 
Modelling 'Twenty Questions'

\subsection{A deep diachronic model for language}

239

In brief, our generative model has to generate a sequence of questions and answers, under the constraint that they are articulated as a discrete sequence of continuous outcomes; here, spoken words. This means that narratives emerge at several (i.e., discrete and continuous) levels, which speaks to the deep or hierarchical aspects of the requisite model. This way of hierarchically framing the conversational dialogue problem, has previously been explored through the inclusion of two separate (fast and slow) levels using artificial neural networks (Serban et al., 2016; Sordoni et al., 2015). To illustrate this deep structure and implicit separation of temporal scales, we considered the problem of generating a succession of question and answers that depend upon beliefs about the world. States of the world come in many flavours. We will refer to these states as hidden factors, where each factor (e.g., 'colour') has a number of discrete states (e.g., 'red', 'green', 'blue' ...). The use of factors is known as a mean field approximation in the variational machine learning literature (Jaakkola and Jordan, 1998; Kschischang et al., 2001; Sallans and Hinton, 2004; Zhang et al., 2018) and is important for simplifying the form of the generative model and ensuing inference. In fact, the notion of approximate Bayesian inference using variational Bayes, is defined operationally in terms of this sort of factorisation.

The problem of specifying a generative model now reduces to specifying the factors that are sufficient to generate a particular question or answer. These include the form of the question, its content, and the beliefs about the world that determine the correct answer. By inducing a factorisation between the form of the sentence and its content, one can finesse the combinatorics of representing all possible questions with all possible content. In other words, we will assume that the brain represents - at some suitably high level - the form of a question and its content separately, where the two only interact when generating an outcome or context for the hierarchical level below.

In this paper, we consider two hierarchical levels; namely, a conceptual level generating syntax and semantics, and a lower level generating lexical sequences of words or phrases. One could consider further levels, all the way down to phonemes and articulation; however, this level of modelling has 
Modelling 'Twenty Questions'

263

already been considered in the context of active listening (Friston et al., under review). We will therefore restrict the current analyses to the generation and understanding of fully formed words (noting that the Matlab simulations that accompany this paper include a full three-level demonstration that supports spoken answers and questions: please see software note).

So, what does one need to know to generate a sentence? Basically, we need the temporal structure or syntax of the question, the semantic content — that fills in content words like nouns and verbs - and the answer (e.g., 'yes' or 'no'). However, to generate syntax and semantics, we need the narrative (e.g., is this a question or answer?) and the form of the question (e.g., is this a question about where something is - i.e., location — or what something is-i.e., shape?). We also need to know the states of the world being described (e.g., contextual or scenic knowledge) and which particular attributes are being discussed. These conceptual constructs constitute the highest level of the generative model; namely, everything one would need to know to specify the syntax and semantics of a lower-level.

In deep models of this sort, deeper hierarchical levels are constituted by factors that change over progressively longer timescales (Friston et al., 2017d; George and Hawkins, 2009; Kiebel et al., 2009). This means higher level factors are attributes of a sentence or phrase, while lower level factors may change from word to word (Davis and Johnsrude, 2003; Specht, 2014). Here, high-level factors include the narrative structure; namely, is this sentence a prompt, question or answer? If this sentence is a question, then what is the question about; e.g., the location or colour of an object? If the narrative requires an answer, then there have to be scenic factors encoding states of the world that render the answer right or wrong; e.g., the object is 'red'. Finally, and possibly most importantly, there have to be factors that support a shared narrative; namely, the shared subject of discussion. We will refer to these as semiotic factors to emphasise that this kind of factor underwrites communication (Roy, 2005; Steels, 2011). In other words, semiotic factors entail latent states that exist only in the context of discourse; e.g., 'we are discussing the colour of something'.

These four kinds of factors (narrative, question, scenic and semiotic) are sufficient to specify a question about something, or an answer, generated under beliefs about something. Crucially, some of these 
Modelling 'Twenty Questions'

289

290

291

292

293

294

295

296

297

298

299

300

301

302

303

304

305

306

307

308

309

310

311

312

313

factors depend upon choices or policies and the others do not. For example, the agent can choose the form of a question and its semiotic content but cannot change scenic states (i.e., the scene or concept being discussed). In addition, we will assume that the narrative cannot be changed, in the sense that a question is always invited by a prompt and is followed by an answer. With this particular construction, agents can update their beliefs about scenic states on the basis of their beliefs about the current semiotic state and responses to questions. In other words, hidden states of the world can be communicated via shared semiotics that rest upon lawful answers to questions under a shared generative model. One can intuit that this generative model requires high-order interactions among the factors in play to generate sentences. In other words, the contingencies that generate a sequence of veridical questions and answers necessarily entail the interactions or conjunctions among several factors. Much of what follows is an attempt to illustrate these interactions using worked examples.

Having specified the form and semiotic content of a sentence, one can now generate a sequence of words in a subordinate level of a generative model that is equipped with probabilistic transitions among lexical states. The implicit transitions from word to word are prescribed by the narrative and question factors of the higher level to generate a syntax, while semantic content is specified by the semiotics. These two attributes (syntactic and semantic) constitute, in the example below, the hidden factors of the lower level of the model. Finally, given these two factors one can generate the appropriate lexical sequence of words; again, via an interaction of (syntactic and semantic) factors.

\subsection{A generative model for "Twenty Questions"}

Figure 2 provides a schematic illustration of this kind of generative model and fills in some of the details (please see figure legend). This example will be used later to illustrate a simplified version of 'Twenty Questions', where a subject has to determine the configuration of two hidden objects by asking a series of closed questions in response to a prompt. The two objects are placed on top of each other and each object can either be a square or a triangle, which can be either red or green. This means that an ideal 
Modelling 'Twenty Questions'

314 (active) Bayesian observer should be able to disclose the configuration with four questions: two

315 questions to establish the colour and shape of the lower object and two questions do the same for the upper object. However, this depends upon asking the right questions, in relation to updated beliefs based upon previous answers. It is this epistemic, uncertainty reducing aspect of communicative exchange we hoped to demonstrate and characterise.

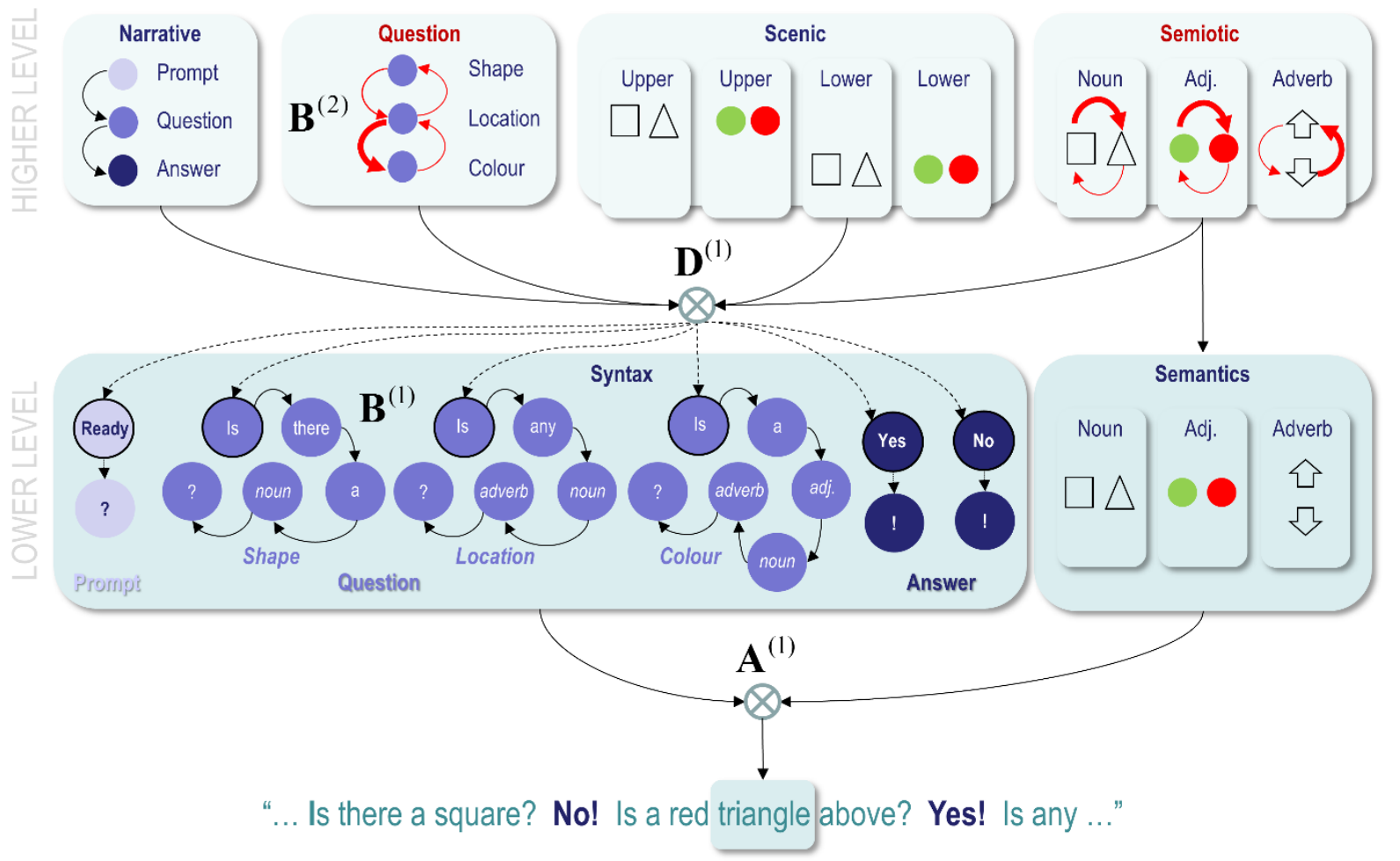

\section{FIGURE 2}

A generative model for Twenty Questions: This figure provides a schematic illustration of the generative model. This schematic displays the architecture involved in generating a sequence of words that could constitute a language-like narrative. In brief, this is a hierarchical (i.e., deep) generative model formulated in terms of discrete hidden states and outcomes (here, outcomes from the lower level are single words). The architecture is deep 
327 because there are two levels of hidden states, where the higher (deeper) level unfolds slowly in time-furnishing 328 contextual constraints on the lower level that generates a sequence of words. The higher level contains hidden 329 factors that generate the syntax and semantic content of a sentence, which are passed to the lower-level. Each 330 panel uses a coloured shape to describe the different states of each factor. At the higher level, transitions among 331 narrative states $\left(\mathbf{B}^{(2)}\right)$ generate a sequence of phrases that cycle in a particular order through "Prompts", 332 "Questions" and "Answers", where their form depends upon interactions with other hidden states in the generative 333 model. The form of questions has been factorised into the type of question ("Shape", "Location", or "Colour") 334 and its semiotic content. The semiotic content has three factors (noun, objective and adverb), each with two states 335 (noun: "square" or "triangle"; adjective: "green" or "red" and adverb: "below" or "above"). Similarly, the four 336 scenic factors correspond to beliefs about the attributes of upper and lower objects in the world; namely, their 337 colours (green or red) and shapes (square or triangle). In this generative model, choices about the type of question 338 and its semiotic content are policy-dependent — as illustrated by the red arrows. In other words, policies determine 339 transitions (encoded by the $\mathbf{B}^{(2)}$ matrices) among controllable states, so that question and semiotic states are 340 selected intentionally. For example, the question generated by the thick red arrows in the figure would be: "Is a 341 red triangle above?" The combination of these states completely determines the syntax and semantic content of a 342 sentence at the lower level (which is encoded in the matrix $\mathbf{D}^{(\mathbf{1})}$ ). The hidden syntax states at the lower level 343 comprise specific words, such as "Ready" and "Is", grammar, such as "?" or "!", and abstract representations, 344 such as noun, adverb, and adjective. The words denoted by the abstract representations are determined by the 345 semantic factor, which is isomorphic with the semiotic factor of the higher level. The first word of the phrase 346 corresponds to the initial syntactic state at the lower level - which is determined by the interactions among states 347 at the higher level, encoded by the mapping D. For example, if the narrative state is a Question, then the initial 348 syntax state is the word "Is", no matter which of the three question states are selected at the higher level. The $\mathrm{B}^{(1)}$ 349 matrices then determine subsequent words (illustrated by the black arrows), by specifying transitions among 350 syntax states that do depend upon the question states at the higher level. However, if the narrative state is Answer, 351 then the initial syntax state can be "Yes" or "No", depending upon high order interactions among the remaining 352 high-level states: a "Yes" will be generated when, and only when, scenic and semiotic states are congruent (e.g., 353 if the question "Is a red triangle above?" admitted a positive response, because a red triangle is in the upper 354 location). For clarity, some syntaxes have been omitted; for example, a "Not sure" answer. In addition, this figure 355 omits embellishments that generate synonymous phrases (e.g., "not sure", "can't say", and so on). The final stage 356 is to map states at the lower level to outcomes at each time step of the generative process. This is denoted by the 357 likelihood mapping $\mathbf{A}^{(1)}$. In the example highlighted here, the articulated word "triangle" depends upon the current 358 syntax state being a noun and the associated content being a "triangle". States without arrows are absorbing states; 359 in other words, the state only transitions to itself. 
Modelling 'Twenty Questions'

362 The particular levels of the factors in the generative model of Figure 2 have been constructed to create 363 a minimal model of how one agent can communicate beliefs about scenic states (i.e., configurations of hidden objects) to another. At the higher level, the model incorporates beliefs about the part of the narrative that is enacted (prompt, question, or answer), the type of question (shape, location, or colour), the putative scenic state of the world, and a semiotic factor indicating the topic of discussion. These factors generate expectations for the lower level: namely, the syntax (i.e., the ordering and content of words) and the semantics (i.e., which shapes, colours, and locations the agent is being questioned on). The lower level thus generates sequences of words, which are concatenated to form phrases—and sequences of phrases (i.e., exchanges) occur as the higher level cycles through the lower level.

Note that the 'syntaxes' included here would not all be considered as syntax under traditional definitions. In the current implementation, syntaxes are just sequences of states (words), with grammar used as terminating states to indicate that the conversational turn has ended.

The repertoire of syntactic structures within this model is limited to three sorts of questions and two sorts of answers; however, even with this limited repertoire, the combinatorics of what could said is non-trivial. To ask a particular question, the subject has to choose the form of the question and the levels of the three semiotic factors by selecting the appropriate policy. To make sense of any answer, it also has to remember these choices. This memory is endowed by a higher level that maintains beliefs about controllable (i.e., question and semiotic) factors, after committing to a particular policy. The selected policy minimises uncertainty and will therefore change with beliefs about the hidden scene, over successive questions and answers, ensuring forward-looking exchanges (Allwood et al., 1992). Note that this kind of working memory-and epistemic behaviour-emerges naturally from maximising model evidence (i.e., minimising variational free energy), given a generative model of successive states of the world that generate outcomes.

We included a prompt state simply to demonstrate the cycling between prompts, questions, and answers. In this formulation, the prompt conveys no interesting information: it is merely part of the structured 
Modelling 'Twenty Questions'

387

dialogue. In the following simulations, we simply use it to convey the type of turn-taking that is often seen in realistic settings.

Clearly, there are many ways in which we could carve up the factors or causes that underwrite linguistic exchanges, and we have ignored many interesting aspects; however, the basic message is that one needs to consider the factorisation and the deep (hierarchical) nature of generative models before dissecting the computational architecture of language. In what follows, we consider more broadly how generative models of this sort can be represented in graphical form, and how variational message passing generates predictions for neuronal dynamics.

\section{Active inference}

The previous section considered the form of a generative model. We can now use active inference to simulate action and perception under that model. The procedures used here assume that the brain restricts itself to a limited number of characteristic states (Friston, 2013) - a property that all sentient systems must possess. Mathematically, these procedures minimise surprise (in information theoretic terms), which is equivalent to maximising Bayesian model evidence; in other words, they maximise the probability of sensory exchanges with the environment, under a generative model of how those sensations were caused. This is the essence of active inference, and implicit self-evidencing (Hohwy, 2016).

Intuitively, self-evidencing means the brain can be described as inferring the causes of sensory samples while, at the same time, soliciting sensations that are the least surprising (e.g., not looking at the sun directly or maintaining thermoreceptor firing within a physiological range). Technically, this take on action and perception can be cast as minimising a proxy for surprise, known as variational free energy. From a statistical perspective, variational free energy can be decomposed into complexity and accuracy, such that minimising variational free energy provides an accurate account of some data in the simplest 
Modelling 'Twenty Questions'

411 way possible (Maisto et al., 2015). Crucially, active inference generalises Bayesian inference, such that

412 the objective is not just to infer the latent or hidden states that cause sensations but to act in a way that

413 minimises expected surprise. In information theory, expected surprise is known as entropy or

414 uncertainty. This means, one can define optimal behaviour as acting to resolve uncertainty: e.g.,

415 saccading to salient, or information rich, regimes of visual space or avoiding outcomes that are, a priori,

416 costly or unattractive. In the same way that direct action and perception minimise free energy, action

417 can be specified in terms of plans or policies that minimise the free energy expected on pursuing that

418 policy.

419 This section briefly reviews parts of active inference that are relevant to the current paper. We begin by

420 explaining expected free energy. We then consider how active inference is applied to discrete generative

421 models, such as the model described in the previous section. Finally, we consider how belief updating

422 can be implemented as a neuronally plausible message passing scheme.

\subsection{Expected free energy}

Expected free energy has a relatively simple form (see Appendix 1), which can be decomposed into an epistemic, information seeking, uncertainty reducing part (intrinsic value) and a pragmatic, goal seeking, cost aversive part (extrinsic value). Formally, the expected free energy for a particular policy $\pi$ can be expressed in terms of posterior beliefs $Q\left(o_{\tau}, s_{\tau}\right)=P\left(o_{\tau} \mid s_{\tau}\right) Q\left(s_{\tau} \mid \pi\right)$ about outcomes and states of the world at time $\tau$ in the future:

430

$$
G(\pi, \tau) \geq-\underbrace{E_{Q}\left[\ln Q\left(s_{\tau} \mid o_{\tau}, \pi\right)-\ln Q\left(s_{\tau} \mid \pi\right)\right]}_{\text {intrinsic value }}-\underbrace{E_{Q}\left[\ln P\left(o_{\tau}\right)\right]}_{\text {extrinsic value }}
$$

431 Extrinsic (i.e., pragmatic) value is simply the expected value of a policy defined in terms of outcomes that are preferred a priori; where the equivalent cost corresponds to Bayesian risk or prior surprise (see 
Modelling 'Twenty Questions'

434 epistemic) value, variously referred to as relative entropy, mutual information, information gain,

435 Bayesian surprise or the value of information expected under a particular policy (Barlow, 1961;

436 Howard, 1966; Itti and Baldi, 2009; Linsker, 1990; Optican and Richmond, 1987). An alternative

437 formulation of expected free energy can be found in Appendix 1: this formulation rearranges the

438 equation for expected free energy, so that it is cast as the expected uncertainty about outcomes (i.e.

439 ambiguity or expected inaccuracy) plus the Kullback-Leibler divergence between predicted and

440 preferred outcomes (i.e., risk or expected complexity). This formulation shows that minimising expected

441 free energy is guaranteed to realise preferred outcomes, while resolving uncertainty about the states of

442 the world generating those outcomes.

443 Here, we are less concerned with the pragmatic aspect of expected free energy and focus on the

444 epistemic drive to reduce uncertainty. We have previously addressed this epistemic affordance in terms

445 of saccadic eye movements - to provide a constructivist explanation for visual searches: c.f., scene

446 construction (Hassabis and Maguire, 2007; Mirza et al., 2016). In this paper, we use a more

447 sophisticated generative model to illustrate the same sort of epistemic foraging, mediated by linguistic

448 exchange. It is worth bearing in mind that the purposeful, inquisitive and abductive behaviours we will

449 see later are all emergent properties of minimising (expected) free energy. In other words, there is no

450 need to handcraft any rules or grammar, or provide any reinforcement or feedback. All of the behaviours

451 shown in this paper result from the structure of the generative model. Subsequent sections will illustrate

452 the belief updating under this model — and so, first, we consider how belief updating relates to neuronal

453 processes and action.

\subsection{Belief updating and neuronal dynamics in discrete generative models}

This section focuses on generative models of discrete outcomes caused by discrete states that cannot be observed directly (i.e., hidden states). This summary is based on (Friston et al., 2017c), which contains 
Modelling 'Twenty Questions'

models correspond to states of the world - that generate outcomes - and the policies that generate successive states. For simplicity, we introduce belief updating in terms of a generic discrete generative model, which has a single level; we then extend this description to discrete hierarchical models containing two levels.

Figure 3 describes the basic form of these generative models in two complementary formats, and the implicit belief updating following the observation of new (sensory) outcomes. The equations on the left specify the generative model in terms of a probability distribution over outcomes, states and policies that can be expressed in terms of marginal densities or factors. These factors are conditional distributions that entail conditional dependencies — encoded by the edges in the Bayesian network on the upper right. The model in Figure 3 generates outcomes in the following way. First, a policy (i.e., a plan or controlled action sequence) is selected at the highest level using a softmax function of the free energy expected under plausible policies. Sequences of hidden states are then generated using the probability transitions specified by the selected policy (encoded in $\mathbf{B}$ matrices). As the policy unfolds, the states generate probabilistic outcomes at each point in time (encoded in A matrices).

The equivalent representation of this graphical model is shown as a Forney factor graph on the lower right. Here, the factors of the generative model (numbers in square boxes) now constitute the nodes and the (probability distribution over the) unknown states are associated with edges. The rules used to construct a factor graph are simple: the edge associated with each variable is connected to the factors in which it participates.

Given a generative model of the form shown in Figure 3, one can construct a neuronally plausible message passing scheme, whose solution minimises free energy. This involves associating the logarithm of an expected hidden state, under a particular policy, with the average transmembrane potential of a neuronal population $\mathbf{v}_{\pi, \tau}=\ln \mathbf{s}_{\pi, \tau}$. By introducing an auxiliary variable (i.e., state prediction error), one obtains the following update scheme, whose solution satisfies the belief update equations in the lower left panel of Figure 3. 


$$
\boldsymbol{\varepsilon}_{\pi, \tau}=\ln \mathbf{B}_{\pi, \tau-1} \mathbf{s}_{\pi, \tau-1}+\ln \mathbf{B}_{\pi, \tau} \cdot \mathbf{s}_{\pi, \tau+1}+\ln \mathbf{A} \cdot o_{\tau}-\ln \mathbf{s}_{\pi, \tau}
$$

$\mathbf{s}_{\pi, \tau}=\sigma\left(\mathbf{v}_{\pi, \tau}\right)$

Generative model

$P\left(o_{1: \tau}, s_{1: \tau}, \pi\right)=P\left(s_{1}\right) P(\pi) \prod_{\tau} P\left(o_{\tau} \mid s_{\tau}\right) P\left(s_{\tau} \mid s_{\tau-1}, \pi\right)$

$1 \quad P\left(o_{\tau} \mid s_{\tau}\right)=\operatorname{Cat}(\mathbf{A})$

$2 P\left(s_{\tau+1} \mid s_{\tau}, \pi\right)=\operatorname{Cat}\left(\mathbf{B}_{\pi, \tau}\right)$

$3 \quad P\left(s_{1}\right)=\operatorname{Cat}(\mathbf{D})$

$4 . \quad P(\pi)=\sigma(-\mathbf{G})$

$Q\left(s_{\tau} \mid \pi\right)=\operatorname{Cat}\left(\mathbf{s}_{\pi, \tau}\right)$

$Q(\pi)=\operatorname{Cat}(\boldsymbol{\pi})$

Approximate posterior
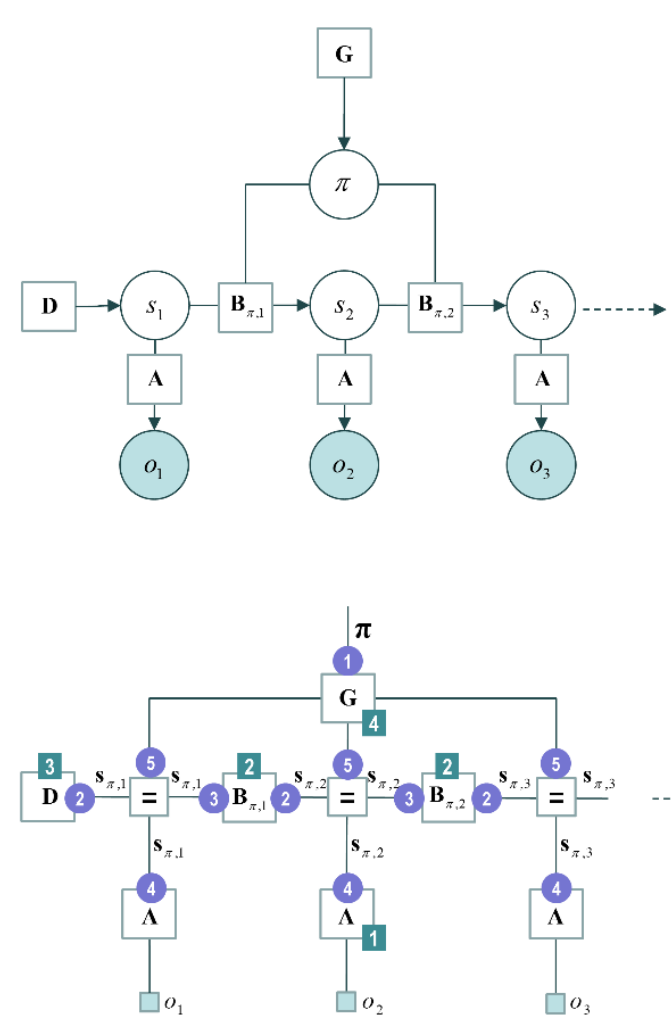

Generative models for discrete states and outcomes. Upper left panel: These equations specify the generative model. A generative model is the joint probability of outcomes and their (latent or hidden) causes, see first equation. Usually, the model is expressed in terms of a likelihood (the probability of consequences given causes) and priors over causes. When a prior depends upon a random variable it is called an empirical prior. Here, the likelihood is specified by a matrix $\mathbf{A}$, whose elements are the probability of an outcome under every combination of hidden states. The empirical priors pertain to probabilistic transitions (in the $\mathbf{B}$ matrix) among hidden states that can depend upon action, which is determined probabilistically by policies (sequences of actions encoded by 
$496 \pi$ ). The key aspect of this generative model is that policies are more probable a priori if they minimise expected

497 free energy G, which depends upon prior preferences about outcomes or costs encoded by $\mathbf{C}$. Finally, the vector

498 D specifies the initial state. This completes the specification of the model in terms of its parameters; namely, A,

499 B, C and D. Bayesian model inversion refers to the inverse mapping from outcomes to causes; i.e., estimating the

500 hidden states that cause outcomes. In approximate Bayesian inference, one specifies the form of an approximate

501 posterior distribution. This particular form in this paper uses a mean field approximation, in which posterior beliefs

502 are approximated by the product of marginal distributions over time points. Subscripts index time (or policy). See

503 Section 2 and Table 1 for a detailed explanation of the variables (italic variables represent hidden states, while

504 bold variables indicate expectations about those states). Upper right panel: This Bayesian network represents the

505 conditional dependencies among hidden states and how they cause outcomes. Open circles are random variables

506 (hidden states and policies) while filled circles denote observable outcomes. Squares indicate fixed or known

507 quantities, such as the model parameters. Lower left panel: these equalities are the belief updates mediating

508 approximate Bayesian inference and outcome selection. When the agent is responsible for generating outcomes

509 (e.g., speaking), they are selected to minimise free energy or, in other words, maximise accuracy under posterior

510 beliefs about the next state of the world. Lower right panel: this is an equivalent representation of the Bayesian

511 network in terms of a Forney or normal style factor graph. Here the nodes (square boxes) correspond to factors

512 and the edges are associated with unknown variables. Filled squares denote observable outcomes. The edges are

513 labelled in terms of the sufficient statistics of their marginal posterior. Factors have been labelled in terms of the

514 parameters encoding the associated probability distributions (on the upper left). The circled numbers correspond

515 to the messages that are passed from nodes to edges (the labels are placed on the edge that carries the message

516 from each node). The key aspect of this graph is that it discloses the messages that contribute to the posterior

517 marginal over hidden states; here, conditioned on each policy. These constitute [forward: 2] messages from

518 representations of the past, [backward: 3] messages from the future and [likelihood: (4] messages from the

519 outcome. Crucially, the past and future are represented at all times so that as new outcomes become available,

520 with the passage of time, more likelihood messages participate in the message passing; thereby providing more

521 informed (approximate) posteriors. This effectively performs online data assimilation (mediated by forwarding

522 messages) that is informed by prior beliefs concerning future outcomes (mediated by backward messages). Please

523 see Table 1 for a definition of the variables in this figure. Adapted with permission from (Friston et al., 2017c). 
Modelling 'Twenty Questions'

526 Although we employ a marginal message passing scheme in the simulations presented later, the 527 derivations presented here use a mean-field approximation to simplify the expressions. While we could 528 have used a mean field approximation and the ensuing variational message passing, this tends to lead 529 to overconfident inferences. Practically, there is little difference between the two: both rely upon the 530 synthesis of local messages from the Markov blankets of variables in the factor graph.

531 These differential equations correspond to a gradient descent on variational free energy as described in 532 (Friston et al., 2017a) and Appendix 2:

$$
\begin{aligned}
\dot{\mathbf{v}}_{\pi, \tau} & =\boldsymbol{\varepsilon}_{\pi, \tau}=-\frac{\partial \mathbf{F}_{\pi}}{\partial \mathbf{s}_{\pi, \tau}} \\
\mathbf{F}_{\pi} & =\sum_{\tau} F(\pi, \tau) \\
F(\pi, \tau) & =\underbrace{E_{Q\left(s_{\tau-1} \mid \pi\right)}\left[D\left[Q\left(s_{\tau} \mid \pi\right) \| P\left(s_{\tau} \mid s_{\tau-1}, \pi\right)\right]\right]}_{\text {complexity }}-\underbrace{E\left[\ln P\left(o_{\tau} \mid s_{\tau}\right)\right]}_{\text {accuracy }}
\end{aligned}
$$

534 Crucially, they also furnish a process theory for neuronal dynamics, in which the sigmoid function can 535 be thought of as a sigmoid (firing rate) activation function of transmembrane potential. This means $\log$ expectations about hidden states can be associated with depolarisation of neuronal populations encoding expectations. This has some construct validity in relation to theoretical proposals and empirical work on evidence accumulation (de Lafuente et al., 2015; Kira et al., 2015) and the neuronal encoding of probabilities (Deneve, 2008). Equivalent updates can be derived for beliefs about policies and the precision of those beliefs. Although omitted from Figure 3 for simplicity, the expected precision of beliefs about policies is interesting because it has all the hallmarks of phasic dopamine dynamics. We will look briefly at simulated dopaminergic firing later. Interested readers are referred to (Friston et al., 2017a; Friston et al., 2014) for details.

544 As noted above, in this (pure communication) setting, outcomes are generated by the agent who is 545 currently speaking. These outcomes are those that minimise variational free energy. These are simply 546 the outcomes that maximise accuracy: 
$o_{\tau+1}=\min _{o} \mathbf{G}_{o}$

$$
\begin{aligned}
\mathbf{G}_{o} & =-E_{Q}\left[\ln P\left(o_{\tau+1} \mid s_{\tau+1}\right)\right] \\
& =-\left(\ln \mathbf{A} \cdot o_{\tau+1}\right) \mathbf{s}_{\tau+1}
\end{aligned}
$$

548 This follows from the fact that the complexity part of free energy does not depend upon outcomes. This 549 sort of outcome is formally related to motor output under active inference; namely, the fulfilment of proprioceptive predictions by classical reflexes (Adams et al., 2013; Shipp et al., 2013). In the current simulations, words or phrases are generated, which play the equivalent role of fulfilling predictions based upon beliefs about hidden states at each point in time.

The final step is to create deep generative models by stacking generative models on top of each other; such that the outcomes generated by one level provide (empirical) priors on the initial states of the level below. By linking hierarchical levels in this fashion, states at the higher level change slowly over time, because states higher level remain the same throughout a sequence of state transitions at the lower level. In the current setting, this means that beliefs about successive words at the lower level are updated on a faster timescale than beliefs about a phrase at the higher level—obliging a phrase to consist of multiple words. Top-down (empirical) priors from the higher level provide a context for inference about the next word, which is informed by all the preceding words in a sentence. This is an important aspect of deep temporal models that lends inference a hierarchical nature; known technically as a semi-Markovian process. Figure 4 illustrates the hierarchical form of the generative model (upper panels) and the accompanying message passing scheme (lower panels) in the form of a factor graph. Note that Figure 4 is simply an extension of Figure 3. At the higher level, the likelihood mapping from hidden states to outcomes (A) from Figure 3 is replaced by a mapping from hidden states in the higher level to the initial states of a lower-level (denoted by D). These mappings allow interactions between states at the higher level to influence states in the lower level. of Twenty Questions in Figure 2. Here, the hidden states have been unpacked into their factors, where controllable states are labelled in red. As noted above, controllable states have transition probabilities 
Modelling 'Twenty Questions'

571 that are prescribed by a policy. For example, for the semiotic factor noun, the available policies could

572 move the semiotic state 'square' to 'triangle'. Crucially, it can be seen that the policy remains in play

573 for a succession of state transitions. In other words, once a policy is inferred or selected, it remains

574 operational in terms of predicting successive outcomes. For example, if I commit to the semiotic state

575 'square', then it remains the subject of the next question and subsequent answer.

576 Equipped with this model and variational message passing scheme, we are now in a position to simulate

577 conversations; both in terms of belief updating and associated neuronal message passing. When the

578 agent is listening, the outcomes can be generated by another agent to simulate dyadic exchange.

579 Conversely, when the agent is talking, outcomes are selected to minimise the free energy under the

580 agent's beliefs. In other words, when the agent is talking, it selects the least surprising words, given its

581 beliefs about the current syntax and semantics. Notice that the agent does not 'know' who is talking-

582 it just expects to hear things that are consistent with its beliefs. If it hears something that is surprising

583 or unexpected, the agent will update its beliefs about the scene and semiotics currently in play. More

584 importantly, the agent's beliefs about what is being said depend upon the policies inferred. These

585 policies minimise expected free energy, which means the agent expects to encounter salient, informative

586 answers and, crucially, questions. In other words, it expects to hear questions and answers that resolve

587 uncertainty, which will be the same as the questions it would ask and the answers it would supply.

588 When considering hierarchical generative models of language processing, we are confronted with the 589 linearization problem (Bornkessel et al., 2005): namely, how are outcomes supplied to higher levels of 590 the generative model and, how do higher levels provide constraints on evidence gathering at lower 591 levels? In other words, how can one accumulate evidence from sequential stimuli to form beliefs about 592 things that do not change with time? Happily, this problem that has already been solved by deep 593 temporal models of the sort above. We demonstrate the implicit message passing and belief updating 594 that underwrites this form of (linearised) evidence accumulation in the next section, by simulating an agent playing the "Twenty Questions" game. 

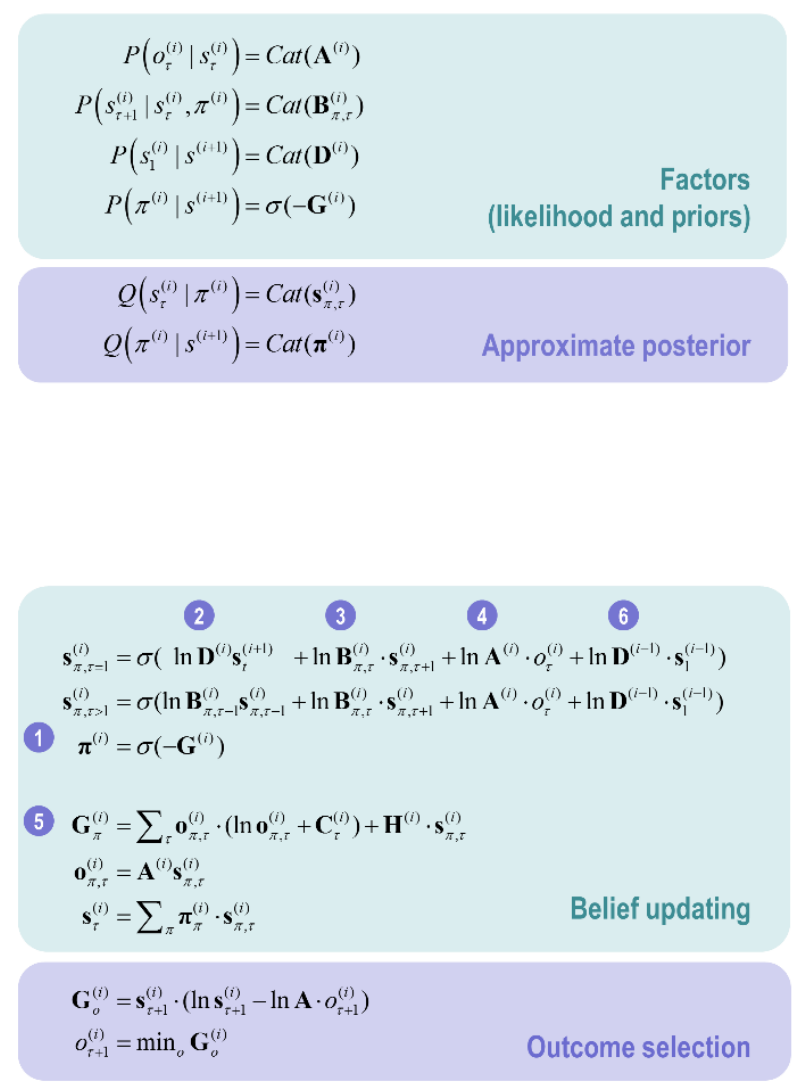

596

597 2017c).
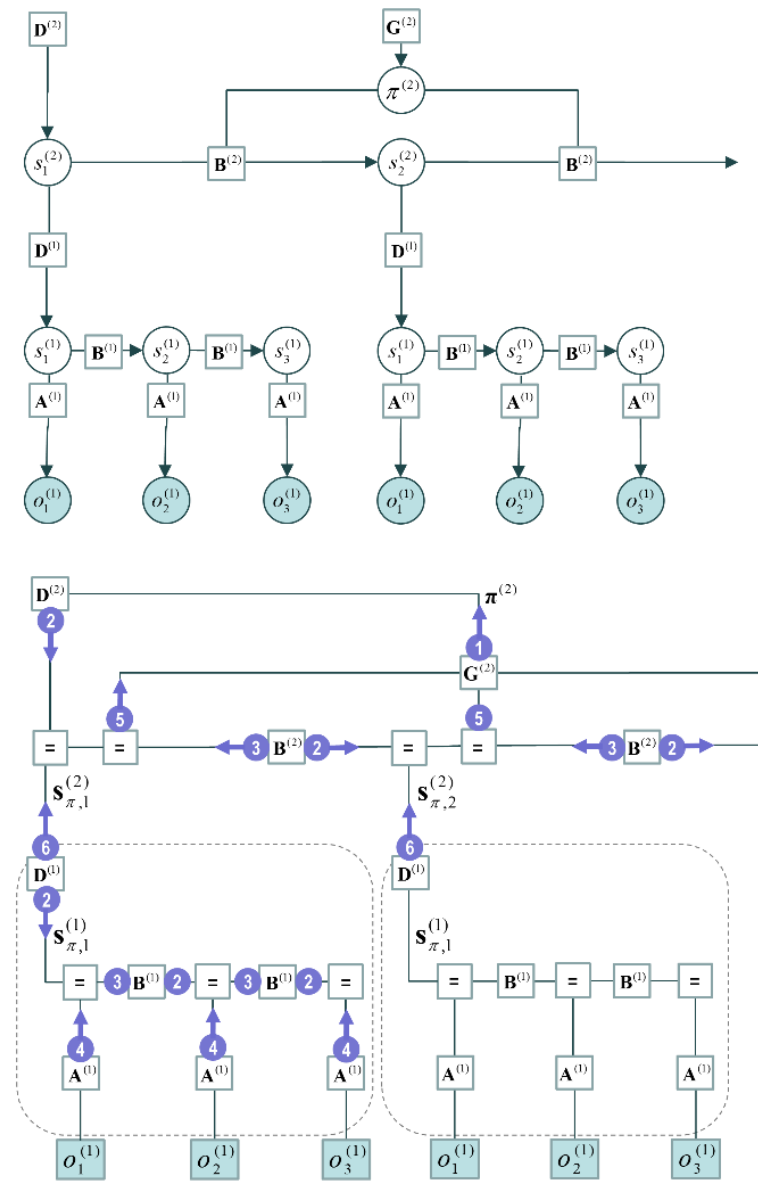

\section{FigurE 4}

Deep temporal models. Left panel: This figure provides the Bayesian network and associated Forney factor graph for deep temporal models, described in terms of factors and belief updates on the left. The graphs adopt the same format as Figure 3; however, here the model has been extended hierarchically, where (bracketed) superscripts index the hierarchical level. The key aspect of this model is its hierarchical structure that represents sequences of hidden states over time or epochs. In this model, hidden states at higher levels generate the initial states for lower levels, which unfold to generate a sequence of outcomes: c.f., associative chaining (Page and Norris, 1998). Crucially, lower levels cycle over a sequence for each transition of the level above. This is indicated by the subgraphs enclosed in dashed boxes, which are 'reused' as higher levels unfold. It is this scheduling that endows the model with deep temporal structure. The probability distribution over initial states is now conditioned on the state (at the current time) of the level above. Practically, this means that $\mathbf{D}$ now becomes a tensor, as opposed to a vector. The messages passed from the corresponding factor node rest on Bayesian model averages that require the expected policies [message $\mathbf{0}$ ] and expected states under each policy. The resulting averages are then used to compose descending [message $\mathbf{2}$ ] and ascending messages [message $\mathbf{6}$ ] that mediate the exchange of empirical priors and posteriors between levels, respectively. Adapted with permission from (Friston et al., 


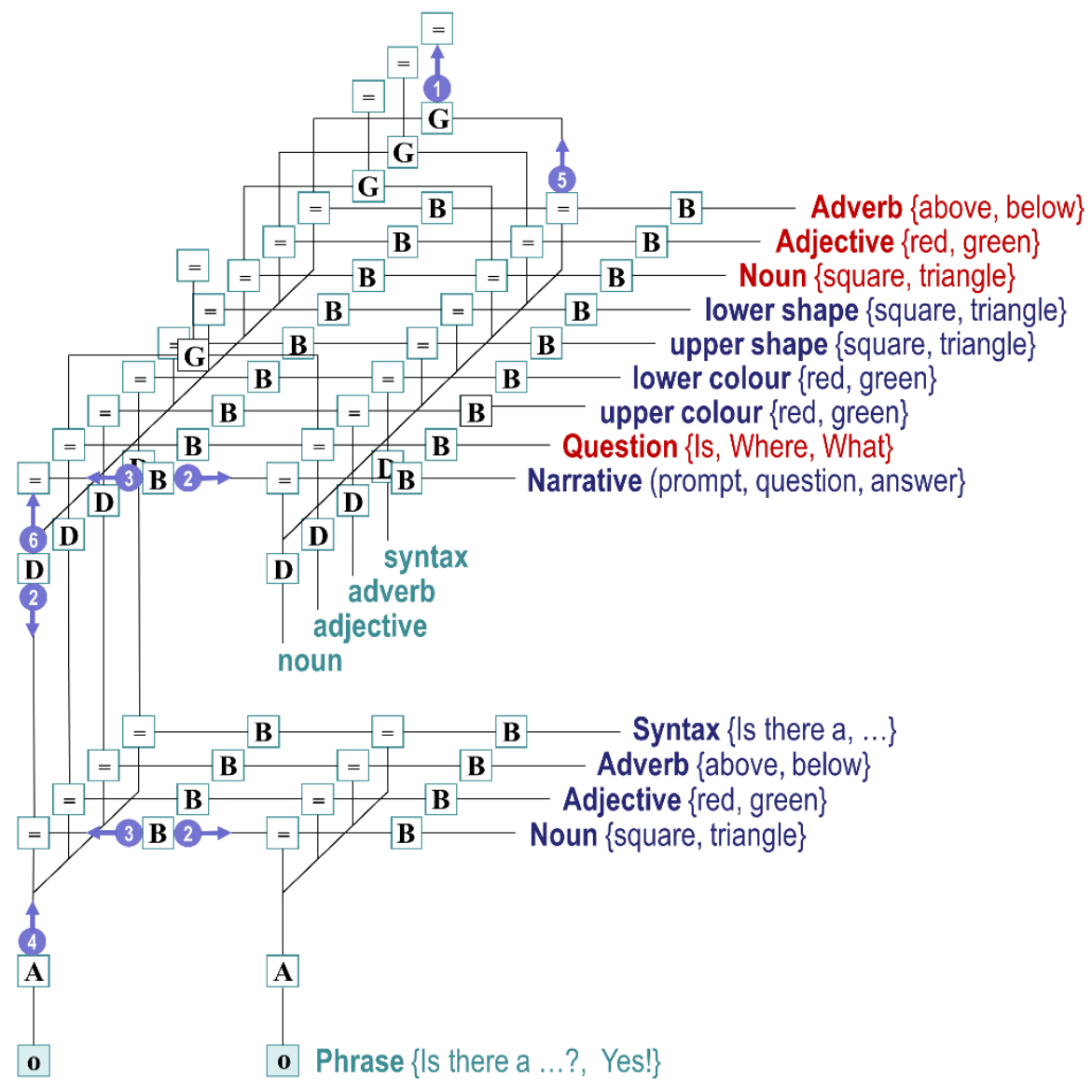

615 Factor graph for $\mathbf{2 0}$ questions: this schematic illustrates the message passing using a Forney style factor graph 616 for the generative model in Figure 2, using the format of Figure 4. In this schematic, we have unpacked the hidden 617 state factors, labelling those with multiple (policy-dependent) probability transition matrices in red. This graphic 618 was produced automatically using the SPM software (please see software note).

\section{4. "Twenty Questions" simulations}

622 To illustrate belief updating — and its neuronal correlates-we use a simplified version of "Twenty

623 Questions". Specifically, we simulated conversations comprising six exchanges, where each exchange

624 comprises three phrases or sentences. The phrases always followed the same sequence: a prompt, a 625 question, then an answer. This order was fixed by specifying very precise priors about transitions among 
Modelling 'Twenty Questions'

626

627

narrative states. Each phrase comprised up to six words, and each word was processed with belief updates described by Equation 3. These updates were evaluated in 16 time-steps of $16 \mathrm{~ms}$ (of simulated and approximately real time). This meant that words were generated every $256 \mathrm{~ms}$, such that a sentence of four words takes about a second to articulate. In these simulations, the artificial agent could take the role of the questioner or the answerer: the agent either listened for the prompt, asked a question, and then listened to the answer, or issued the prompt, listened for a question, then supplied the answer. In all cases, the agent (slightly) preferred affirmative answers ("Yes") over negative answers ("No"). These preferences were specified by setting prior costs of $\mathbf{C}=-1 / 4$ for "Yes" and $\mathbf{C}=1 / 4$ for "No" (see Table 1). This means that the agent will ask questions that it believes will elicit a "Yes" answer, everything else being equal.

In these simulations, the agent started out with uniform prior beliefs about which colour and shape was present at the two locations (above and below). It played the role of the questioner for the first four exchanges, after which it identified the colours and shapes of both objects with high confidence. Having updated its beliefs, it then switched roles to answer two questions. To allow the agent to play the roles of the questioner and answerer for these simulations, we separated the agent's generative model from the generative process; effectively, this means that the agent was 'in conversation' with the generative process. The generative process had exactly the same form as the generative model, except the generative process had more precise beliefs about the scene. Here, as the agent was not equipped with beliefs about whether it was speaking or listening, we simulated turn-taking by sampling the output from the agent or generative process at the appropriate stages of the exchange.

Figure 6 displays the results of belief updating. Each panel shows posterior expectations about the two hidden objects after each of the six questions were answered. The questions are shown in black text towards the top of the panel, while the answer is shown at the bottom of the panel. All answers in this example were correct (i.e., correspond to the true scene), so are displayed in green. Within each panel, the agent's beliefs about shape (square versus triangle) and colour (green versus red) at the two locations are depicted with large icons. The true (narrative) scene is shown with small icons to the right. 


\section{Behavioural responses and underlying beliefs}
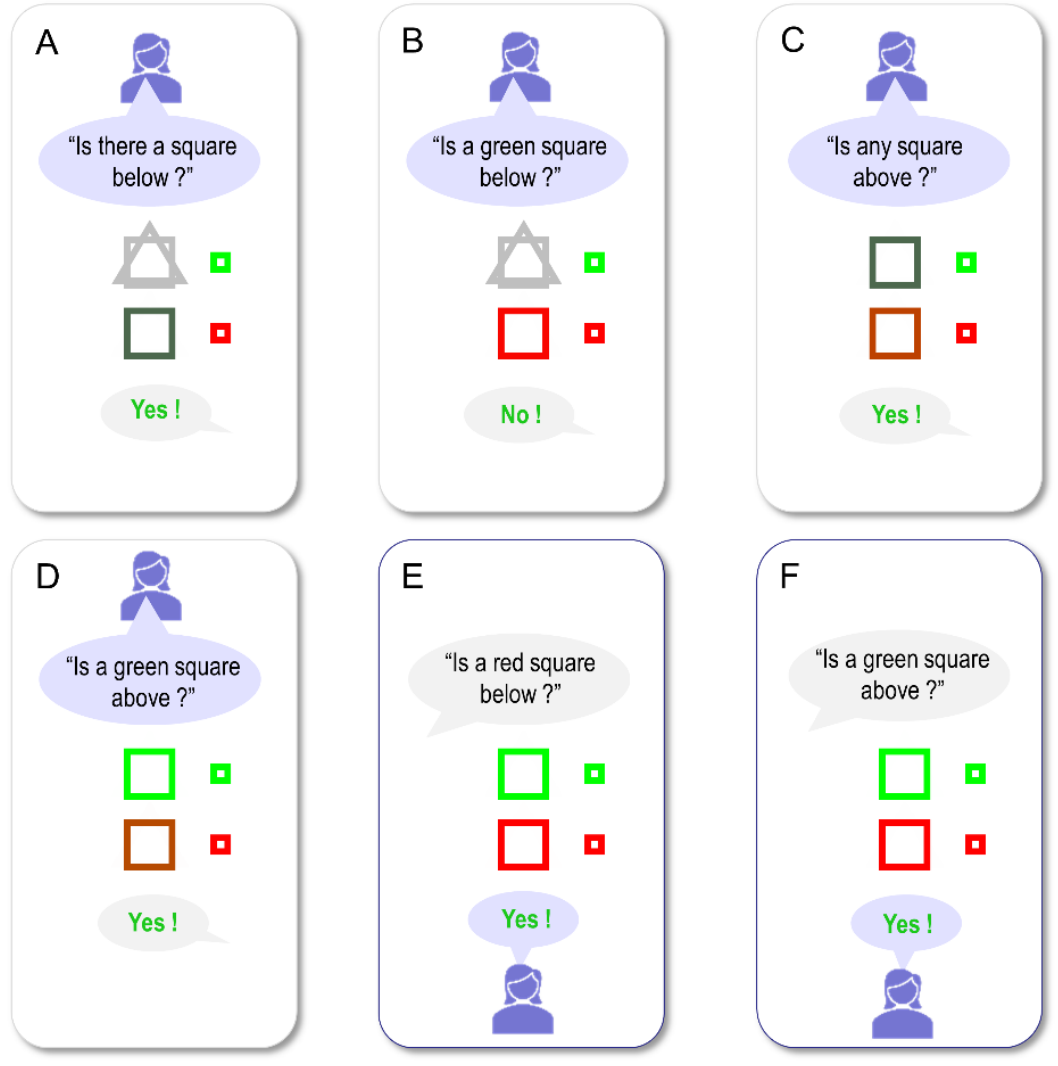

652

654 Behavioural responses: Each panel shows the posterior expectations of a synthetic subject after its question had

655 been answered. The agent's beliefs about shape (square versus triangle) and colour (green versus red) for the

656 upper and lower locations are depicted with large icons. Where the agent has no particular (i.e., uniform) beliefs,

657 the two shapes are displayed overlaid and/or in grey (e.g., upper locations in panels A and B); where the agent's

658 beliefs tend toward a particular colour, the shape is shaded slightly red or green. The true scene (with veridical

659 colours and shapes) is shown with small icons to the right. The question is shown in black text (above each set of

660 expectations), while the answer is shown below. All of the answers in this simulation are correct, so they are

661 displayed in green text. The human icons and purple callouts are positioned next to the agent's vocalisations, to

662 illustrate whether the subject was asking questions (first four exchanges) or answering them (last two exchanges). 
Modelling 'Twenty Questions'

665 During the first four questions, the agent accumulates evidence and builds veridical beliefs about the scene at hand. At the beginning, it has no particular (i.e. uniform) beliefs about the shapes and colours at the two locations. First, it chooses to ask a question about the shape, because it is more likely to get an affirmative (preferred) response than if it were to ask a question about shape and colour together. After the first answer, it knows there is a square below (see first panel) and subsequently asks a question about the combination of shape and colour. After the second answer (see second panel), it knows that the square is not green and must therefore be red. It then goes on to ask similar questions for the upper location, after which time it holds precise beliefs about the shapes and colours at the two locations. By the time it answers the fifth and sixth questions, the agent can provide veridical answers to questions about specific scene components (the fact these responses are in green text indicates that the answers are correct).

Notice that the expectations of the colour (red) of the lower panel become less precise after first inferring there is a red square below (compare second and third panels). This arises because we have slowed down belief updating, so that its time constants correspond roughly to those observed empirically (see below). This precludes complete convergence to free energy minima during belief updating. The ensuing uncertainty is then carried over to the next exchange. Further, notice that after responding correctly to the question about the colour of the lower square (fifth question; lower middle panel), the agent's beliefs are refreshed as the answer provides confirmatory evidence about what was believed.

As anticipated, the artificial agent resolved uncertainty about the hidden scene after only four questions, suggesting that appropriate questions were asked. For example, the first question establishes that there is a square below, while the second discloses the fact that it is red. It could have opted to ask only What questions, but then it could have needed as many as 8 questions to infer the correct scene. Notice also that the second question is not redundant: it is asked in the context of knowledge that the lower object is square. A possible second question would have been to ask: "Is there a circle below?", but given (i) the agent already knows the lower object is a square and (ii) in this scenario only one object is present at each location, this question would not reduce uncertainty about the contents of the scene. Ultimately, 
Modelling 'Twenty Questions'

691 the behaviour demonstrated in these simulations emerges because the agent selected policies that reduced uncertainty about the scene. This can only happen because the generative model entertains future states, which enable the agent to evaluate expected outcomes in the future. For example, any answer to the second question ("Is a red square below") completely resolves uncertainty about its colour. The agent knows this question will resolve uncertainty before the question is even asked. Thus, this type of question has epistemic value.

697 Note the subtle nature of this epistemic behaviour: the agent is using semiotics states (noun, adjective, and adverb), over which it (believes it) has control, to resolve uncertainty about scenic states, over which it (believes it) has no control. In this scenario, the agent exerts control by generating outcomes (e.g., questions); it will generate outcomes that are the least surprising, under uncertainty resolving policies. This vicarious belief updating is central to the current formulation when considering how we might install beliefs in others through linguistic communication.

\subsection{Message passing and neurophysiology}

Having illustrated belief updating behaviour, we now take a closer look at the predictions of this type of inference, or sequential evidence accumulation, for neurophysiology. Figure 7 illustrates electrophysiological and dopaminergic responses to the six questions from the simulation above. These responses are shown in various formats: Figure 7A shows posterior expectations about the colour of the lower object at various times during the sequence of six narratives, displayed in raster plot format. On this plot, each prompt, question, and answer, is assumed to last for $250 \mathrm{~ms}$. For simplicity, this figure displays only the colour states. Different units are labelled on the Y-axis; namely, green or red at successive epochs $(1,2$, and 3$)$ within each exchange.

713 During the time window corresponding to the first question and answer ( $0-0.75$ seconds), Figure $7 \mathrm{~A}$ is shaded in grey, indicating that the agent has uniform beliefs about the colour of the object during the

715 first question, which queries the shape and not colour. The second question asks about the colour of the 
Modelling 'Twenty Questions'

716 lower object. This question Figure 6B has the answer "No" (i.e., not green), indicating that the colour

717 of the square at the lower position must be red. The plot shows that this answer induces profound belief 718 updating; the belief that the lower square is red is very precise and this belief is maintained (i.e., 719 'remembered') throughout the exchange (i.e., for the remainder of the time plotted). During this time, 720 the shading on this plot allows us to visualise the reduction in precision for the belief that the object is 721 red, and subsequent reinstatement of precision after the fifth question — as discussed in the previous 722 section.

723 Notice in Figure 7A that the latency of this belief updating for expectations at the beginning of the trial 724 is greater than at the end - this is due to message-passing backwards in time (message 3 in Figure 4). 725 As noted above, these posterior beliefs decay a little over subsequent trials, until the agent reaffirms its conviction that the lower colour is indeed red.

727 Figure 7B shows the same data in a different format. Here, pooled expectations (after filtering between 7284 and $32 \mathrm{~Hz}$ ) are shown as a white line. This is superimposed upon a time-frequency heat map to 729 illustrate bursts of frequency-specific energy during periods of belief updating. We will examine this 730 characterisation in more detail later, with reference to the next figure.

731 Figure 7C illustrates the simulated fluctuations in neuronal activity, after bandpass filtering. These can 732 be regarded as simulated local field potentials or event related potentials (Leonard et al.), corresponding 733 roughly to the voltage fluctuations in Equation 3. Later in the paper, we will revisit these synthetic ERPs 734 to characterise responses to surprising outcomes. The current simulation simply shows that the 735 amplitude of simulated ERPs is related to the amount of information conveyed by an outcome - it shows 736 greater responses to more informative parts of the narrative. 
A

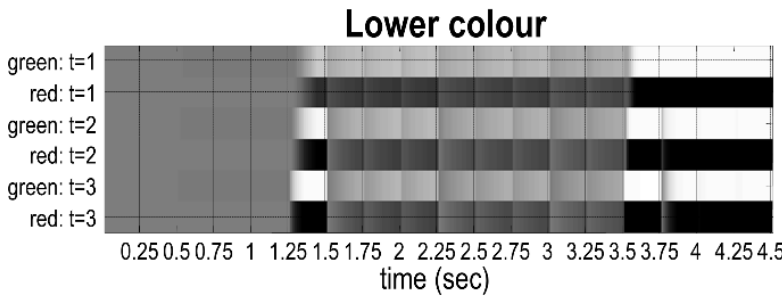

B

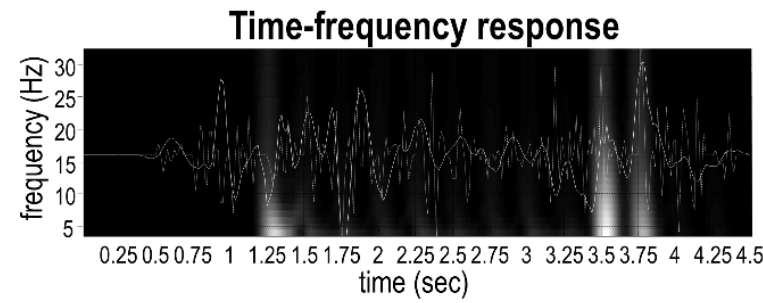

C

Local field potentials

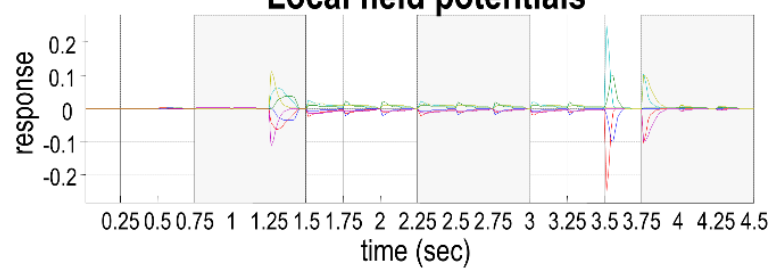

D

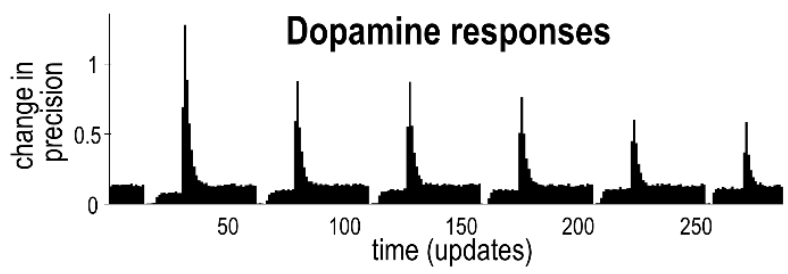

739 Electrophysiological responses: This figure shows the simulated electrophysiological responses associated with

740 the belief updating reported in Figure 6. In this figure, we focus on beliefs about the colour of the lower object,

741 which is at the higher level of the generative model — thus, these plots show simulated responses following each

742 phrase (i.e., prompt, question, and answer) rather than following each word. The horizontal axes show time over

743 the entire exchange, assuming each phrase lasts for $250 \mathrm{~ms}$. Expectations about the hidden state encoding the

744 colour of the lower object are presented in raster format in the panel A, where black corresponds to maximum

745 firing rates. Panel B shows the same data but in a different format: here, pooled expectations (filtered between 4

746 and $32 \mathrm{~Hz}$ ) are shown as a white line. This simulated local field potential is superimposed upon a time-frequency

747 heat map to illustrate bursts of frequency-specific energy (white), during periods of belief updating. The

748 underlying fluctuations in simulated neuronal activity, after bandpass filtering between $4 \mathrm{~Hz}$ and $32 \mathrm{~Hz}$, are shown

749 in panel C. Each of the coloured lines on this plot represent belief updating for a given unit (i.e., the rows of the

750 upper panel). Panel D shows simulated dopamine responses after each answer: these attenuate as uncertainty is

751 resolved. 
Modelling 'Twenty Questions'

752 Finally, Figure 7B shows simulated dopamine responses (i.e., expected precision of beliefs about policies), as described in (Friston et al., 2014). Interestingly, the peaks of these phasic responses coincide with times that answers are given. The key point to take from these phasic responses is that the implicit changes in confidence — about the policies being pursued — depends on the extent to which answers resolve uncertainty and fulfil prior preferences. Every time the agent receives (and to a lesser extent delivers) an answer, it becomes more confident about what it is doing. However, becoming more confident about the hidden scene attenuates the 'confidence boosts' (i.e., phasic dopamine responses). Anecdotally, this seems consistent with the subjective experience of "Twenty Questions", where each confirmatory answer is rewarding, especially at the beginning of the game.

Figure 8 presents the simulated electrophysiological responses from Figure 7 in terms of what one would predict when analysing spectral responses from the higher order area during belief updating. The lower panels show the spectral responses. The lower left panel reports the log spectral density of the six units (i.e., neuronal populations), whose event related responses are shown in Figure 7C. This shows that spectral responses show a degree of scale-free broadband activity, reflecting the fact that the simulated neuronal dynamics have multiple nested timescales.

The ensuing nonlinear coupling between fluctuations at different frequencies is summarised in terms of cross frequency coupling on the lower right panel of Figure 8. This simple characterisation is the correlation between the response magnitudes, over frequencies ranging from 4 to $32 \mathrm{~Hz}$ (based on the time frequency response in the lower panel). The key thing to note from this panel is the off-diagonal structure, suggesting that there are profound correlations among different frequencies. This would be interpreted as some form of nonlinear or phase-amplitude coupling, if subject to standard procedures such as bi-coherence analysis or phase-synchronisation measures: for example, see (Giraud and Poeppel, 2012; Lizarazu et al., 2019; Pefkou et al., 2017). In general, this nonlinear coupling is consistent with "evidence that the temporal modulation transfer function (TMTF) of human auditory perception is not simply low-pass in nature" (from (Edwards and Chang, 2013) p113). 


\section{Spectral responses and nested oscillations}

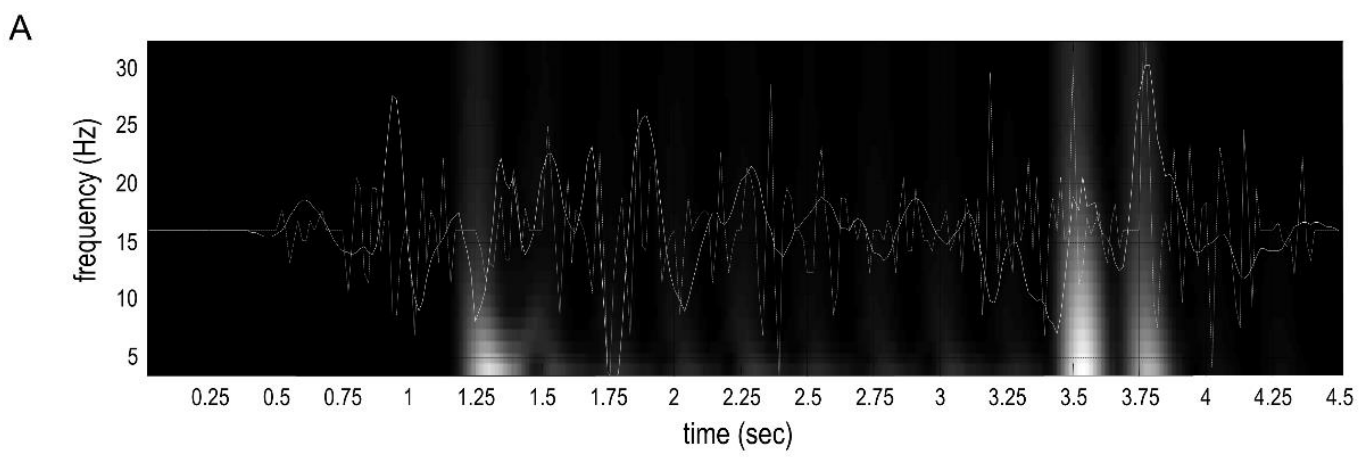

B

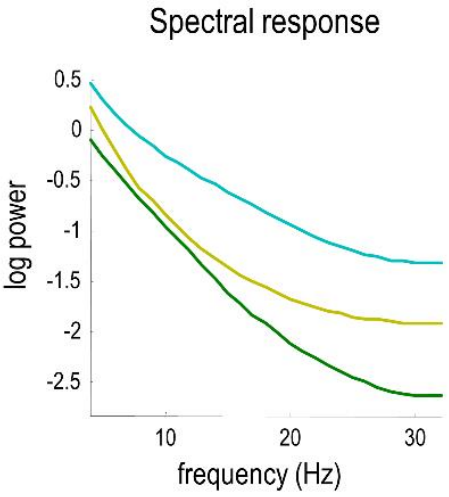

C Cross-frequency coupling

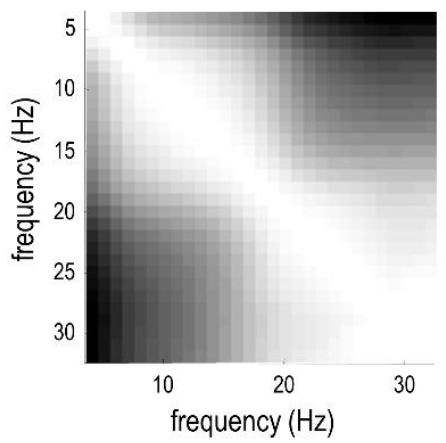

\section{FIGURE 8}

779 Spectral responses and nested oscillations. This figure shows the spectral responses associated with the 780 simulated electrophysiological responses in Figure 7. Panel A is a reproduction of Figure 7B. Panel B reports the

781 spectral density of the six units (i.e., 'red' or 'green' for epochs 1,2, and 3). Only three lines are visible because pairs of responses overlap perfectly. Note that the scale is expressed in terms of log power. The matrix in panel C shows the correlation between the magnitudes of responses over frequencies ranging from 4 to $32 \mathrm{~Hz}$. These correlations are based on the time frequency response in panel A. 
Modelling 'Twenty Questions'

786 The implicit nonlinear coupling arises, in this setting, from the message passing within and between

787 hierarchical levels of deep temporal models. Neuronal dynamics perform a gradient descent on

788 variational free energy, as each new outcome becomes available (for the higher level, when each phrase

789 is spoken). By virtue of this temporal scheduling, there are necessarily nested oscillations in the sense

790 that fast (e.g., gamma) fluctuations unfold at a slow (e.g., theta) rhythm (Friston et al., 2017a): a

791 succession of transients containing high-frequency components is induced by hearing each word, and

792 these transients recur at the (necessarily lower) frequency of word presentation.

793 In short, these types of responses would be expected, based on belief updating at different hierarchical

794 levels of a generative model, which necessarily occur at different temporal scales. This nesting is

795 illustrated in Figure 9, which shows the simulated neuronal firing and associated local field potentials

796 for neuronal populations at the higher and lower levels. The key thing to observe here is that lower level

797 transients (cyan lines) are faster than the accompanying higher-level transients (red lines). This means

798 that fluctuations in the amplitude of frequency-specific responses to each word or phrase will

799 necessarily produce cross frequency coupling. Phenomenologically, this means that one would not be

800 surprised to see bursts of beta activity at the higher level coincide with bursts of gamma activity in the

801 lower-level. See (Arnal and Giraud, 2012; Giraud and Poeppel, 2012) for a discussion of related

802 phenomena. 

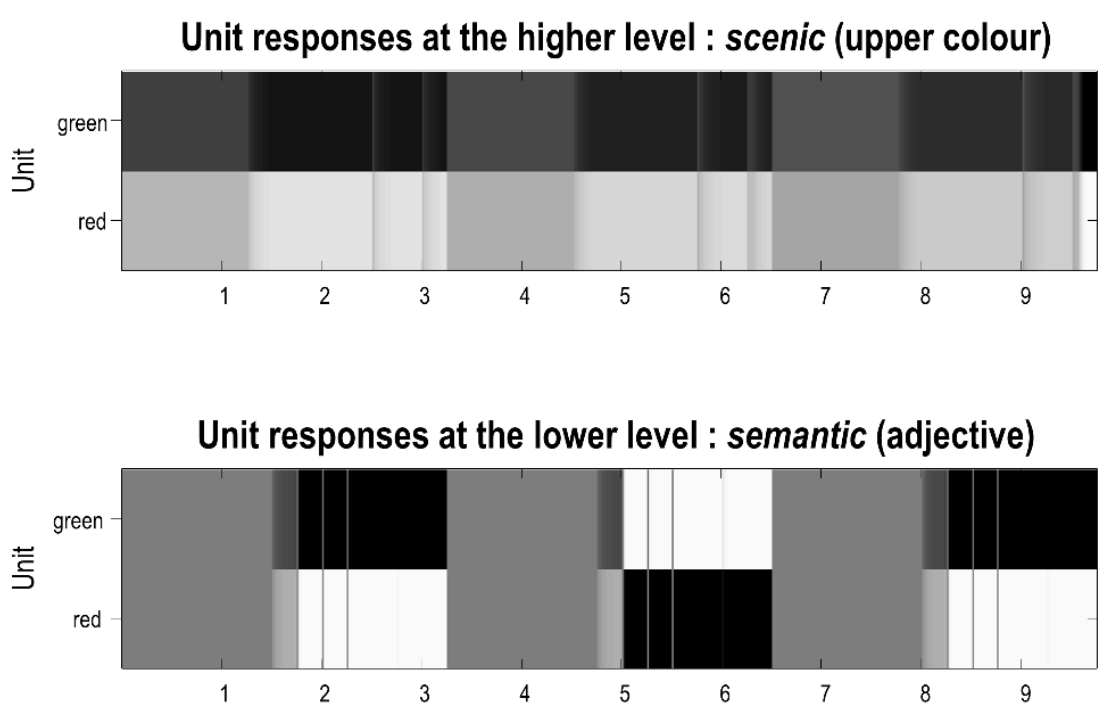

Local field potentials

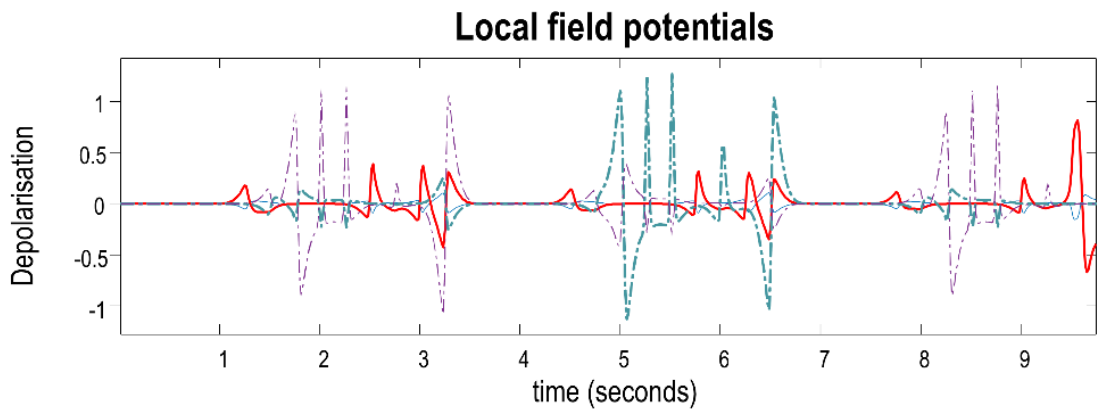

Hierarchical message passing and nested oscillations. The upper panel illustrates responses at the second level using the format of the upper panel of Figure 7. Here, we focus on representations of the colour of the upper object - following each phrase - for the last three exchanges. At this point, the agent is fairly sure the upper object is green (as indicated by the darker shading for the 'green' unit in the upper panel. The middle panel shows the equivalent results for representations in the lower level, encoding the semantic adjective factor, which switches between green and red for the last three questions. The lower panel shows the band-pass filtered responses (between 4 and $32 \mathrm{~Hz}$ ) to illustrate the nesting of simulated electrophysiological responses (solid lines: higher-level scenic responses. Broken lines: lower-level semantic responses). Two responses have been highlighted for illustration in red (high level) and cyan (lower level). The nesting of (simulated) neuronal fluctuations is evident at a number of levels. First, bursts of activity are organised around periods of belief updating, when sensory evidence becomes available. Periods of activity are evoked by auditory outcomes (words) at the lower level and - at the higher level — evidence that speaks to the posterior expectations or representations. Second, it demonstrates transients at the onset of each word, which recur at a theta frequency. Each transient carries fast (e.g., gamma) frequency components. This means there is a theta-gamma coupling in the sense that the amplitude of gamma responses fluctuates at a theta frequency. Finally, note that the transients at the lower level (cyan line) are 'sharper' than the transients at the higher level (red line). 
Modelling 'Twenty Questions'

\subsection{Deep violation responses}

824 Thus far, we have focused on the sort of message passing — and its neurophysiological correlates - that would be measured using time frequency analyses of induced responses. Here, we consider predictions for evoked responses. In particular, we simulate the differential waveforms evoked by stimuli that violate expectations, which have been the focus of many empirical studies; e.g., (Coulson et al., 1998; Friederici, 2011; Pulvermuller et al., 1995; Van Petten and Luka, 2012; Ylinen et al., 2016).

Figure 10 illustrates the neurophysiological simulation of a violation response; for example, P300 or N400 responses to a semantic violation or unexpected sentence closure. Here, we reproduce a violation paradigm by rerunning the fifth exchange from the previous simulations with the wrong answer at the end. Recall that at the beginning of fifth exchange, the agent is confident that the colour of the lower shape is red: it obtained this information from the answer to question 2. The left panels show the standard responses, using a similar format to Figure 7.

Simulated event related potentials (i.e., band pass filtered expectations of the lower colour at the three epochs) are shown on the upper right. The underlying unit activities producing these fluctuations are shown in terms of a simulated raster of unit firing — for the six units in question—on the lower left. Of note, simulated event related potentials in the right panel (i.e., when the wrong answer is provided at 0.5 seconds) have longer latencies (as illustrated by the blue arrow). These long latency responses have a remarkably similar morphology to P300 (Donchin and Coles, 1988; Van Petten and Luka, 2012) and N400 (Kutas and Hillyard, 1984; Van Petten et al., 1999; Van Petten and Kutas, 1990) waveform components in empirical violation paradigms. Here, they simply reflect the fact that the artificial agent has to change its mind and undo the conviction that the lower square was red, given the evidence was in favour of green; in other words, update its beliefs. As can be seen from the lower right panel, the agent becomes less certain about the colour, and leans slightly towards the belief that the colour of the lower object is green. This posterior belief is entirely congruent with hearing a negative answer to the question "is there a red square below?". 


\section{Neuronal violation responses}
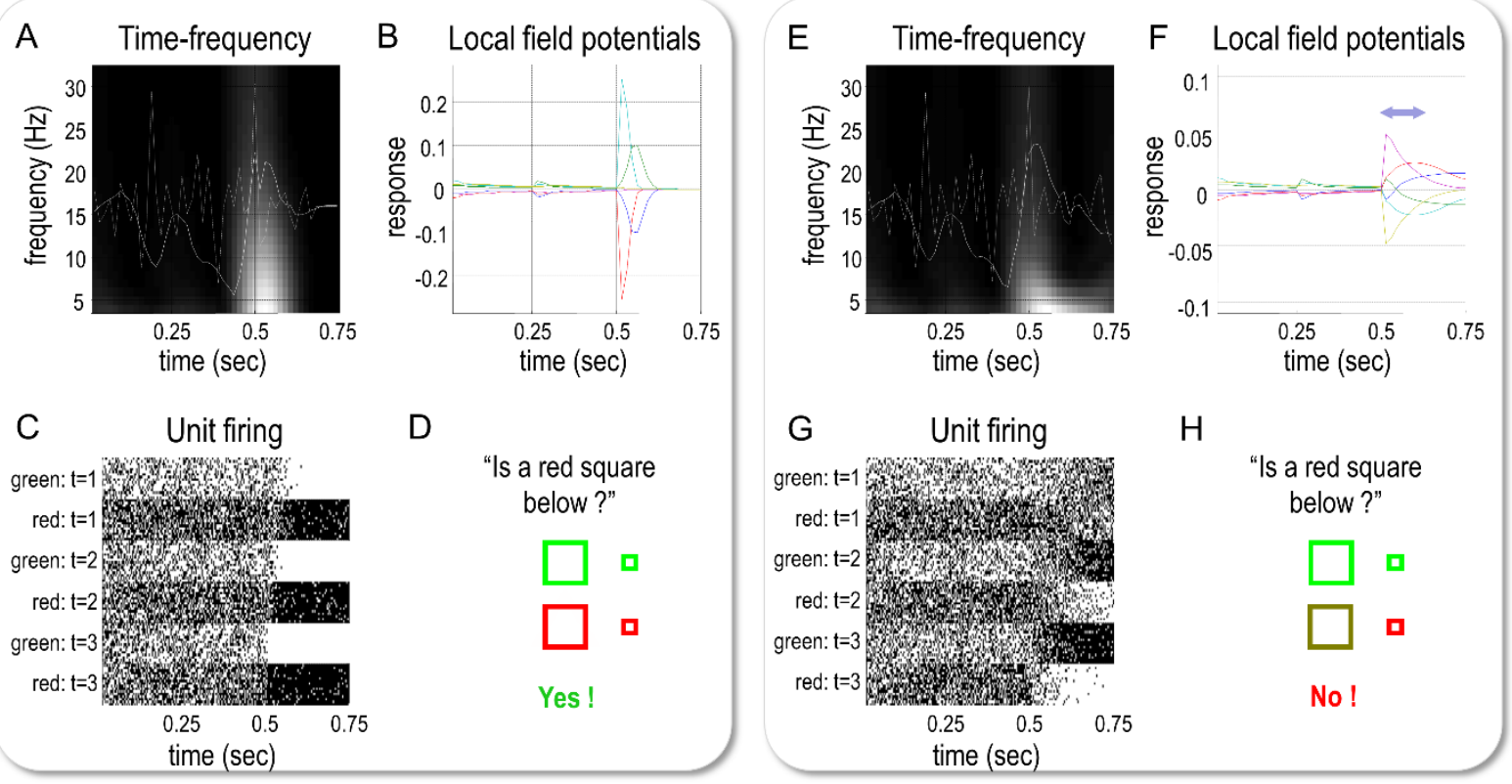

850 Violation responses: This figure illustrates the neurophysiological simulation of a violation response, of the sort seen in response to a semantic violation or unexpected sentence closure. We reproduced this paradigm by rerunning the fifth narrative but supplying the wrong answer at the end (see panel $\mathrm{H})$. The left box (A-D) shows the standard responses when the correct answer is supplied (see panel D) using a similar format to Figure 7. Here, the simulated unit firing of neurons that respond to the colour of the lower object (i.e., the scenic representation at the higher level) are shown in raster format (panel C). The population average or expected firing rate is used to simulate unit activity by sampling from a binomial distribution at each $16 \mathrm{~ms}$ time window. The average response magnitude and time frequency response are shown in panel A for the three epochs (prompt, question, answer) of the fifth exchange. The simulated event-related potentials (i.e., expectations about the colour of the lower object-red or green - at the three epochs, band pass filtered at 4-32 Hz) are shown in panel B. The right box $(\mathrm{E}-\mathrm{H})$ reproduces the same results after supplying the wrong answer (i.e., "No" versus "Yes"), which induces protracted belief updating over a longer latency, as indicated by the blue arrow. 


\section{Synthetic communication}

867 In the simulations above, external states of affairs were used to supply veridical answers to the first four questions by sampling from the generative process. In other words, the external states were standing in for the beliefs of someone answering or asking questions. In what follows, we make an important move and replace external states with another synthetic subject. This has the interesting consequence of taking external states off the table: outcomes are generated or sampled from the (posterior) predictions of one or other subject, so at no point do we need to refer to (external) states of the world (see Figure 1). This is a straightforward consequence of allowing agents to generate outcomes that are shared between them. Heuristically, the imperative to resolve uncertainty (i.e., minimise expected free energy) is now reflected in a synchronisation of belief states; namely, a 'meeting of minds' and mutual understanding.

\subsection{Questions and answers}

The simulations reported in Figure 11 use a similar format to Figure 6 however, here there are two synthetic subjects. The second subject has precise (i.e., confident) beliefs about the scene at hand (namely, a green square above and a red square below). In contrast, the first subject is less confident before the exchange begins and effectively inherits the belief of the confident subject by listening to the answers to the questions it asks. Analogous to Figure 6, after the fourth answer, the first subject has a precise understanding of what the confident subject believes and is able to answer correctly the when quizzed with two final questions.

In this example, the first agent chose its questions carefully to resolve as much uncertainty as possible. In the lower panel, we reverse the roles so that the second (confident) agent asks questions and the less confident agent provides answers. The results of the simulation are shown in the lower panel (entitled role reversal). In comparison to the upper panel, the first agent accumulates evidence for the beliefs of the second agent much more slowly, and the two agents do not share the same beliefs after the fourth answer. The questions asked by the second agent are insensitive to the particular uncertainty that 
Modelling 'Twenty Questions'

891 confounds the first, and so all the first agent can do is say that it is "not sure" in response to the first two questions, when its beliefs are uniform across the red and green states. For the third question, it responds "no". Unlike the first two questions, the third question asks about the combination of colour and shape attributes in the upper location, which has four possible options, and so the balance of probabilities means that the most likely answer is "no". After hearing its own negative answer, the subject then convinces itself that the upper shape cannot be a red triangle and is more likely to be a green square, which is further endorsed by its subsequent response to the (same) fourth question. Only when observing definitive and veridical answers can it then start to accumulate proper beliefs about what the other subject believes.

\subsection{Storytelling}

902 We can use exactly the same scheme above to simulate instruction or storytelling: the same underlying joint belief updating characterises all forms of exchange in this active inference formulation. We reran the simulations from the previous section, but this time the second agent answered its own questions, while the first simply listened for the first four exchanges and supplied answers for the last two exchanges. As above, the first agent inherits scenic beliefs from the second agent, but here this is simply

907 by listening to the second agent's soliloquy. After the four questions and answers, the first agent is

908 sufficiently confident about the scene to answer correctly; even though it is unsure whether the lower 909 object is a red square or a red triangle. This ambiguity reflects the fact that the preceding questions and 910 answers were not selected to reduce the first agent's uncertainty-they were selected by the second 911 subject, who had very precise beliefs. 
Twenty Questions
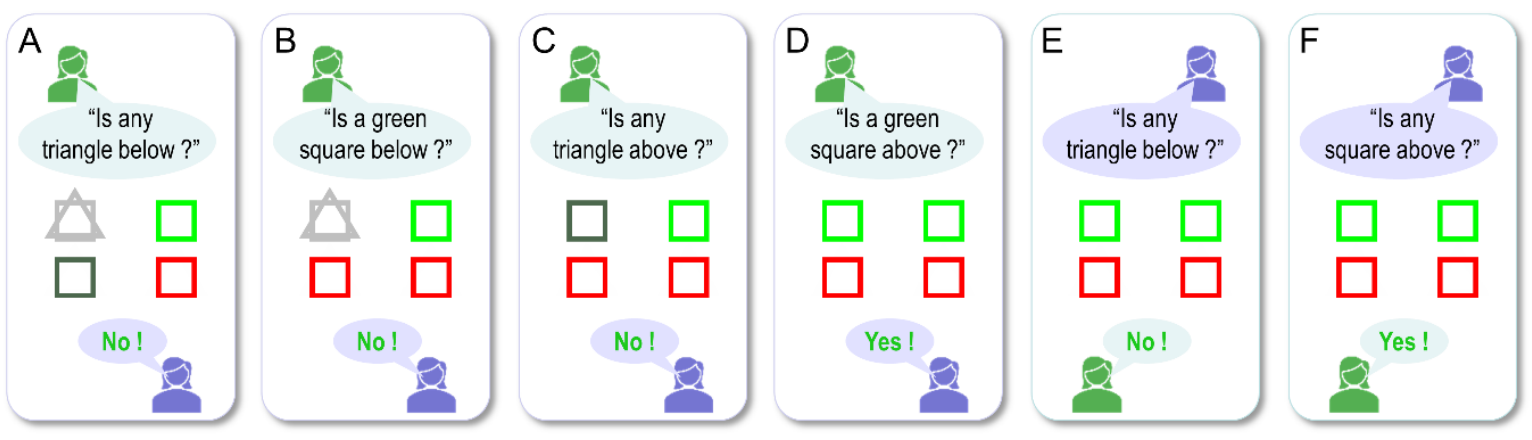

\section{Role reversal}
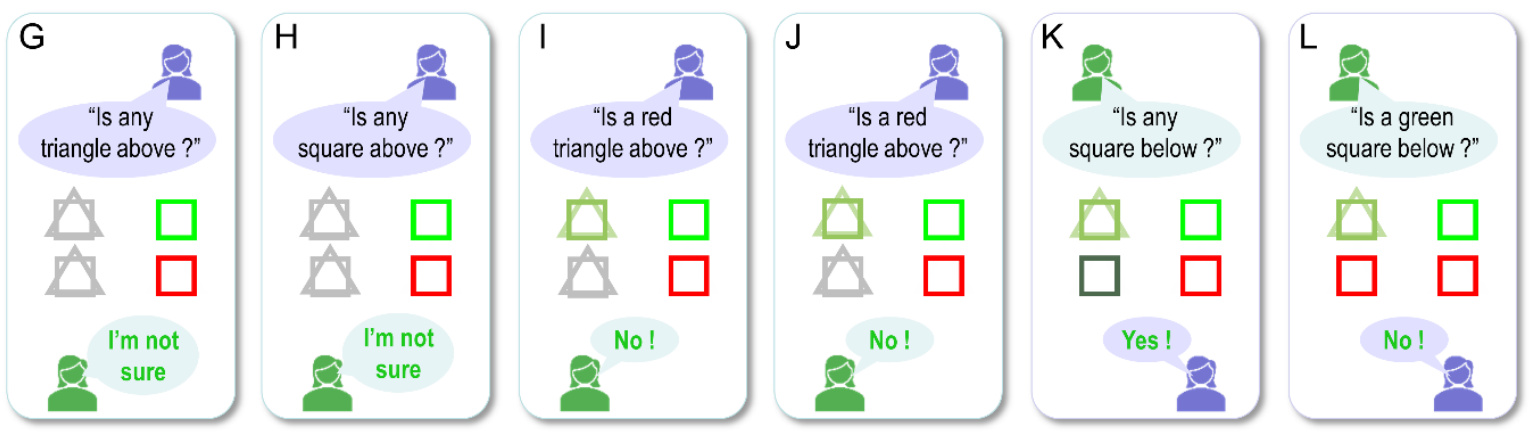

914 Playing 'Twenty Questions' with a partner: These simulations use a similar format to Figure 6;

915 however, here there are two synthetic subjects. Their beliefs are displayed in separate columns within

916 each panel, and the text is placed next to the agent who spoke the phrase. The second subject (purple

917 icon, right column) has precise (i.e., confident) beliefs about the scene at hand: it believes there is a

918 green square above a red square). In contrast, the first agent (green icon, left column) begins with

919 imprecise beliefs and effectively inherits the beliefs of the confident subject, by listening to the

920 answers to the questions it asks. It is then able to answer the two questions asked by the other agent in

921 the fifth and sixth narratives. The lower panels replicate the simulation but here the less confident

922 agent answers questions. 


\section{Telling a story}
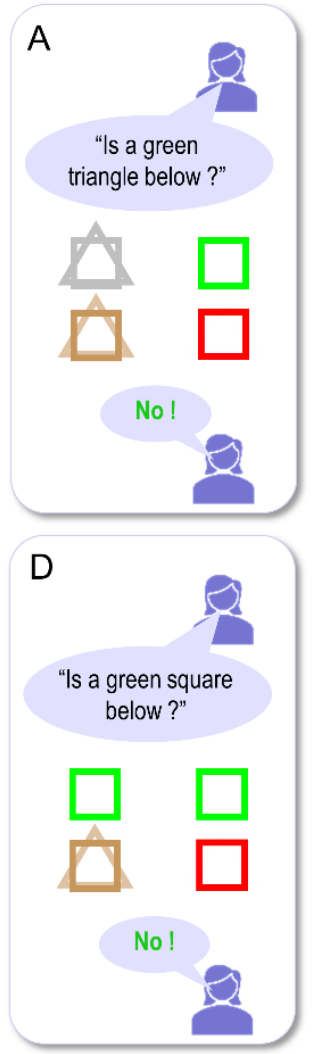

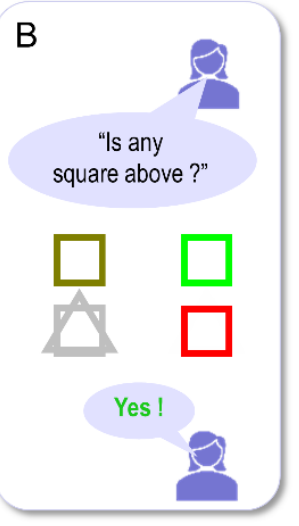

$\mathrm{E}$

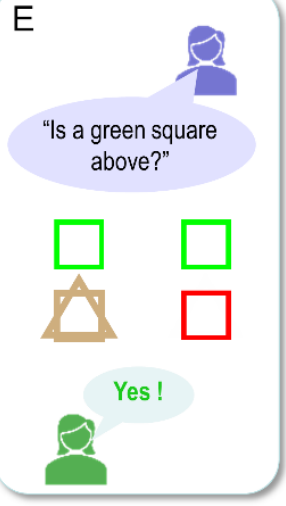

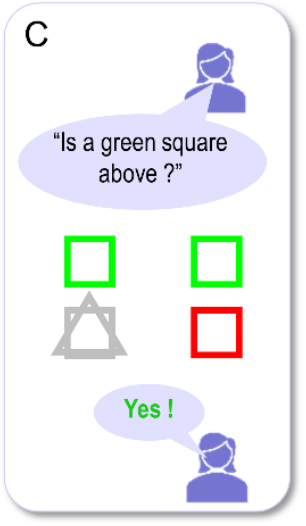

$\mathrm{F}$

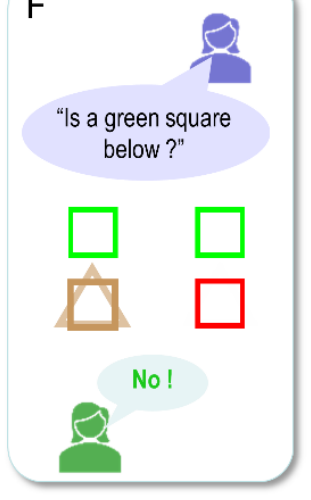

925 Storytelling: The result of an exchange between two synthetic agents, when the second agent (purple icon, right

926 panel) answered its own questions for the first four exchanges (panels A-D). For the fourth and fifth exchanges 927 (panels E-F), the second agent asked the questions and the first agent (green icon, left column) answered. Here, 928 the first agent had to rely upon the question selected by the second agent to update its beliefs about the scene. This 929 resulted in some residual ambiguity about the lower object (i.e., it is most likely to be a red triangle, it could be a 930 red square, but it is probably not a green square). Nevertheless, the first subject was still able to answer the 931 questions correctly. 
Modelling 'Twenty Questions'

\subsection{Making your mind up}

933 Things get interesting if we reduce the precision of prior beliefs, so that both subjects are uncertain

934 about the scene. Recall that the synthetic agents are given three possible responses: "Yes!", "No!", and

935 "I'm not sure". When allowed to question each other in this setting, they simply respond truthfully that

936 they are unsure about the answer (see the upper panel of Figure 13). However, when we reduce the prior

937 probability of the 'not sure' response, both subjects effectively tell each other about what they believe,

938 until they come to hold the same beliefs (see the lower panel of Figure 13). At this point, uncertainty is

939 precluded because each can predict the other and their shared understanding. This is an example of

940 neural hermeneutics (Frith and Wentzer, 2013) in the absence of 'truth pointing'. As noted above, this

941 is a form of generalised synchronisation (Friston and Frith, 2015a), where the orbits of belief states that

942 underlie linguistic exchange become mutually predictable as (expected) free energy is minimised.

943 Anthropomorphically speaking, the two synthetic subjects have simply reached a consensus about how

944 to describe some shared construct. Crucially, the construct (i.e., scene) does not exist and, from our

945 perspective, therefore, could be described as a Folie à deux (Arnone et al., 2006). On a more positive

946 note, it could also be construed as a joint exercise in creative thinking. Although not pursued here, one

947 can think about extensions of this sort of simulation that could be framed in terms of artistic

948 communication and creativity, bringing us back to the resolution of uncertainty through epistemic

949 foraging, novelty and fun (Schmidhuber, 2006).

950 Returning to the upper panel of Figure 13, this example illustrates the joint maintenance of uncertain

951 beliefs. This is interesting because these rudimentary agents have no formal metacognitive capacity (see

952 discussion). In other words, their uncertainty is implicit in neuronal states encoding uncertain belief

953 distributions, rather than possessing neuronal states that encode posterior beliefs about the precision of

954 their beliefs. Having beliefs about the precision of beliefs may sound rather complicated; however,

955 statistical models with a deep structure very often encode uncertainty explicitly. For example, when we

956 report the degrees of freedom of a statistical test, we are effectively reporting the confidence in our

957 estimate of uncertainty; e.g., the standard error on some parameter estimates (Friston et al., 2007). In 


\section{No one knows}
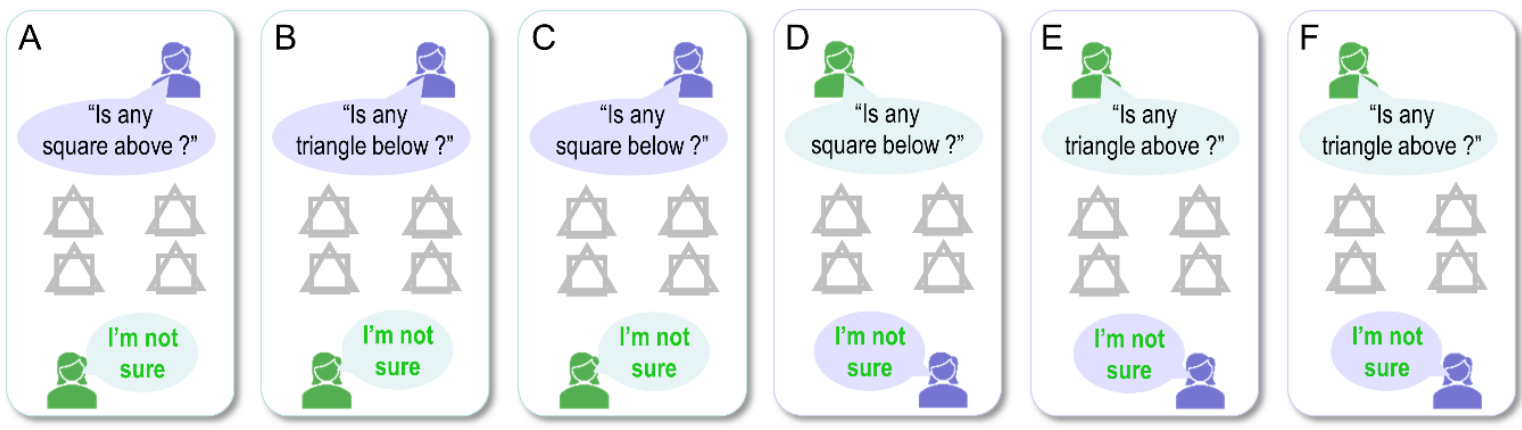

\section{A shared narrative}
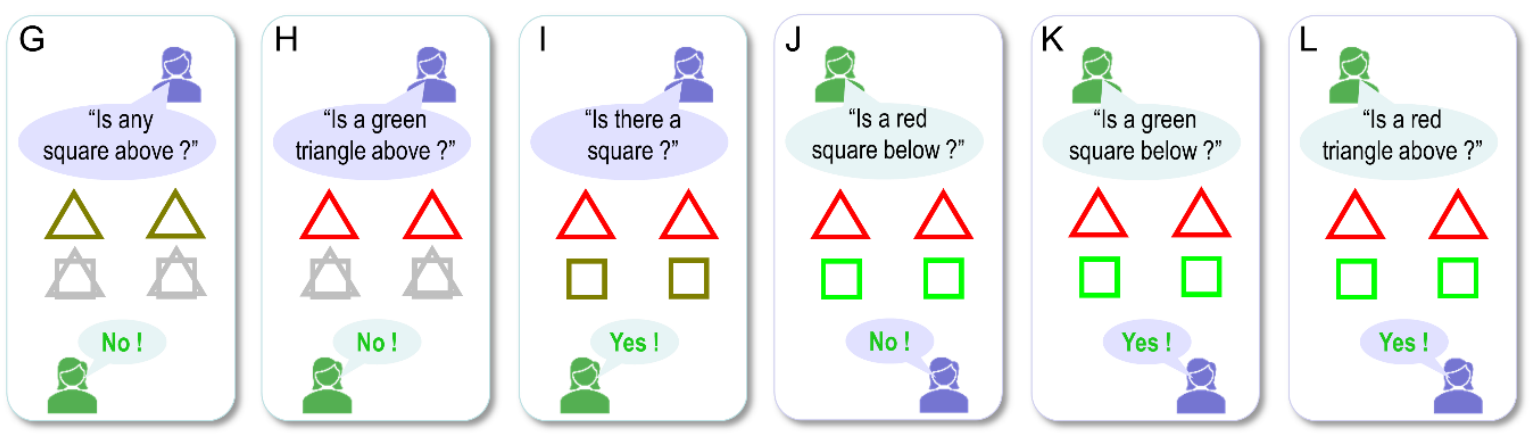

962

FigURE 13

Folie à deux: The result of an exchange between two interlocutors (green and purple), who are both unsure about the scene they are discussing. The format of this figure follows that of previous figures. The upper panels (A-F) show the questions and answers that confess a lack of knowledge or certainty. Each agent's posterior expectations about the scene are indicated by the coloured shapes. In this simulation, neither agent informs the other agent about the objects present in the scene, and so they both remain in a state of mutually consistent ignorance. The lower panels (G-L) show the same simulation when the likelihood of an "I'm not sure" response was set to zero.

970 This produces a folie à deux described in the main text. In brief, the ensuing belief updating starts from an unstable fixed point of uncertainty that converges onto a shared fantasy about what both agents (are confident they) believe. 
Modelling 'Twenty Questions'

972 The mechanism that underwrites this apparent confirmation of 'known unknowns' is straightforward.

973 It rests upon a nontrivial likelihood of saying "I'm not sure", irrespective of one's beliefs. Consider the

974 following: I am thinking about numbers between one and one hundred and I can either report a number

975 or select a "not sure" option. If the likelihood of reporting a number is $90 \%$ and I am sure about the

976 number, then I am nine times more likely to report the (exact) number I have in mind than to say "not

977 sure". Conversely, if I have no idea about the number, then the likelihood of reporting any number is

978 equal to the probability of selecting any other number; the probability of reporting any individual

979 number therefore falls to less than $1 \%$, because the probabilities are dispersed or diluted over 100

980 number options. In this case, I am therefore more than 10 times more likely to report "not sure" than

981 any individual number. In Bayesian model selection, this phenomena is known as evidence dilution

982 (Hoeting et al., 1999). The example in the upper panel of Figure 13 highlights this emergent but simple

983 consequence of entertaining declarations of uncertainty. Note that this kind of uncertainty rests upon a

984 shared generative model, in which uninformative responses can be selected, even in the absence of

985 uncertainty. When we remove the opportunity to generate such agnostic responses, a different pattern

986 of mutual understanding emerges (see the lower panel of Figure 13).

987

988 6. Discussion

989 In summary, we have illustrated a number of plausible correlates of communication that emerge from

990 active inference under a particular sort of generative model. This generative model was motivated by

991 the nature of language and communication. The key attributes of this model speak to the notion of a

992 shared narrative that reduces uncertainty. In the Section 4 (“Twenty Questions" simulations), we

993 simulated an agent that was speaking to itself (i.e., in 'conversation' with a generative process), and in

994 the Section 5 (Synthetic communication) we used exactly the same generative model to simulate two

995 subjects who were asking and answering questions. In each of these cases, the inference and sensory

996 evidence were identical: the only difference was agency (i.e., who was talking). 
Modelling 'Twenty Questions'

997 In our simulations, hierarchical inference led to belief updating that resembled theta-gamma coupling

998 (Figure 8), which has often been observed empirically; for example, see (Giraud and Poeppel, 2012;

999 Lizarazu et al., 2019; Pefkou et al., 2017). Under this framework, theta-gamma coupling arises because

1000 hearing each word induces a succession of transients containing high-frequency (gamma) components,

1001 and these transients recur at the frequency of word presentation, which is in the theta range. A multi-

1002 timescale nesting process has been proposed by others as a plausible explanation (Arnal and Giraud,

1003 2012; Giraud and Poeppel, 2012), as it has been noted that the timing of these rhythms corresponds to

1004 important timescales in language. Previous approaches to modelling this phenomenon (Hovsepyan et

1005 al., 2018; Hyafil and Cernak, 2015) have been data-driven-incorporating explicit theta and gamma

1006 'units'. Here, we took a theoretical approach and show that theta-gamma coupling can arise from belief

1007 updating, given an agent's goal to understand the contents of a scene from a dialogue.

1008 Our simulations also predict electrophysiological violation responses, of the sorts observed in P300 and

1009 N400 studies. The P300 has been observed in oddball paradigms, in which repeating stimuli are

1010 interspersed with unexpected deviants. In this setting, the P300 has been interpreted as reflecting

1011 violations of high-level context (Donchin and Coles, 1988). The N400 is commonly observed in studies

1012 of language. It has been elicited when participants hear words that have low frequency (Kutas and

1013 Hillyard, 1984; Van Petten et al., 1999; Van Petten and Kutas, 1990), or words that are semantically

1014 related to words that have high probability (Kutas and Hillyard, 1984).

1015 The generative model we have introduced represents a different way to think about semantic or contextual aspects of language, in relation to previous accounts. Surveying the empirical and theoretical

1017 antecedents of the current formulation of language — and understanding — would be an enormous

1018 undertaking, given the vast amount of psychological, philosophical and computational literature in this

1019 area. In this context, three observations are relevant. First, in the current framework, belief updating is

1020 hierarchical: beliefs about the content of a scene are maintained at the higher level. Second, an agent's 
Modelling 'Twenty Questions'

1022 Finally, here, we cast language understanding as an active processes - allowing an agent to ask questions that maximally resolve their uncertainty about states of affairs.

1024 Before commenting upon some salient points of contact with related work, we will qualify this 1025 discussion with the following observations: if one commits to active inference (and implicitly, the free 1026 energy principle), there is little latitude for hypothesising about the nature and form of linguistic 1027 processing. This is because everything of interest is defined operationally by the generative model and 1028 the generative model is, in turn, defined by what we want to explain; namely language and understanding. In other words, simply defining the inference problem dictates the form of the requisite generative model, in terms of what how outcomes are caused by states of the world (or others).

1031 Furthermore, once the generative model has been specified, the belief updating is prescribed by standard 1032 belief updating schemes; here, variational or marginal message passing (Dauwels, 2007; Parr et al., 1033 2019; Winn and Bishop, 2005).

This means that there is no latitude to accommodate alternative hypotheses or constructs, if they are not consistent with the sort of formulation above — or the basic architecture of belief updating. In short, in active inference, the only questions are: what kind of generative model could explain these responses?

1037 Strictly speaking, this precludes questions about the implementation and the neurophysiological correlates of language processing (Braiman et al., 2018; Dowty et al., 1985; Lizarazu et al., 2019; Pefkou et al., 2017; Wilson et al., 2017; Ylinen et al., 2016). While many of these may be especially useful within their own remit, unless neurophysiological correlates can be linked to belief updating (i.e., understanding through communication), they cannot be used to simulate — and therefore understandcommunication. In a similar vein, any exciting advances in computational neurolinguistics (Barlow, 1974; Lightfoot, 1979; MacKay and Peto, 1995; Norris et al., 2016; Rosenfeld, 2000) that do not deal 1044 explicitly with belief states updating cannot be used to create artefacts that communicate. For example, 1045 the use of deep learning in speech recognition may provide compelling insights into the computational architecture of linguistic processing at an auditory level; however, speech recognition does not constitute understanding. In other words, simply mapping from auditory input to a list of words does 
Modelling 'Twenty Questions'

1048

1049

1050

1051

1052

1053

1054

1055

1056

1057

1058

1059

1060

1061

1062

1063

1064

1065

1066

1067

1068

1069

1070

not constitute the inversion of a generative model. Some research, within machine learning, has looked at schemes similar to active inference, within partial observability frameworks. For example, the Bayesian Action Decoder (Foerster et al., 2018) uses approximate Bayesian update to obtain a 'public' belief that is conditioned on the actions of all agents in the environment, leading to efficient communication when playing multi-agent games.

There are important developments in computational linguistics that could inform active inference schemes in a useful way. For example, the use of hierarchical Dirichlet processes to solve the structure learning problem in generative models of language (MacKay and Peto, 1995; Salakhutdinov et al., 2013) could be the right approach to grow generative models - and subsequently prune them with Bayesian model reduction (Friston and Penny, 2011) - in the context of language acquisition. We have not touched upon this issue in the current paper; however, having established the basic form of a generative model for language and understanding, the next challenge would be to study learning through optimisation of the model parameters; e.g., the likelihood mapping is entailed by the A matrices between hierarchical levels. After this (learning) has been addressed, the next level of optimisation concerns the form and structure of the model itself. For example, how many hidden factors should be included—and how many levels or mutually exclusive states occupy each factor? This is the problem of structure learning (Catal et al., 2019; Gershman, 2017; Tenenbaum et al., 2011; Tervo et al., 2016) that is elegantly addressed using nonparametric Bayesian methods (Collins and Frank, 2013; Goldwater, 2006; Teh et al., 2006), such as those found in computational linguistics (please see below).

In this paper, we ignored the attribution of (i.e., inference about) agency; namely, metacognitive capability (Fleming et al., 2012; Shea et al., 2014). This means that each synthetic subject had no notion of who was talking. Nevertheless, our synthetic subject could still use the information provided to resolve uncertainty about states the world (e.g., the configuration of objects in a scene). More sophisticated generative models would include hidden factors that include agency per se. This was not necessary for the current examples, but would be necessary for simulating turn taking in linguistic exchange (Garrod and Pickering, 2009; Ghazanfar and Takahashi, 2014; Wilson and Wilson, 2005). 
Modelling 'Twenty Questions'

1074 This was a focus of our earlier work using simulated songbirds (Friston and Frith, 2015a). In the current

1075

1076

1077

1078

1079

1080

1081

1082

1083

1084

1085

1086

1087

1088

1089

1090

1091

1092

1093

1094

1095

1096

1097

1098

1099 work, we simply replaced internally generated speech with the external speech of a conversant to simulate asking questions and answering, respectively. However, the agents were not aware of this.

An important aspect of metacognition is knowing when one is uncertain. In the simulations above, agents were able to maintain their uncertainty by providing each other with uninformative ("not sure") answers. However, they were not aware of being uncertain (i.e., their generative models did not have a hidden 'state of uncertainty'). A more sophisticated generative model would realise that something was not known with confidence and respond with "I really don't know". This apparently simple capacity rests upon a generative model of confidence that is quintessentially metacognitive; in the sense that inverting this kind of deep generative model produces (posterior) beliefs about beliefs.

It is an interesting challenge to formulate metacognitive depth using discrete state space models (i.e., hidden Markov models or Markov decision processes). In one sense, the encoding of precision or confidence in beliefs about policies is a metacognitive representation (see the simulated dopamine responses in Figure 7); however, it is quite elemental. Furthermore, this sort of representation is a continuous (real valued) variable, of the sort that has been used to explain dopaminergic fluctuations in reinforcement learning paradigms (Schwartenbeck et al., 2015). It would be nice to have the categorical step state of "I am uncertain" or "I am very confused". This speaks to the use of higher hierarchical levels that prescribe uniform (empirical) priors over the initial states of a level below. In other words, one can generate belief distributions about the context of a lower level, based upon a discretisation into confident beliefs about particular states of affairs and complete uncertainty (with uniform priors). In principle, this should equip agents with a metacognitive sense of their beliefs-and a way of communicating these beliefs via language.

An important aspect of language that we ignored is its computational richness (e.g., discrete infinity) afforded by the combinatorics of narratives and sentences (Chomsky, 2017). In addition, we have ignored the parsing and transpositions that characterise real language processing - that themselves have a deep hierarchical form. This issue presents some interesting challenges, in terms of articulating the 
Modelling 'Twenty Questions'

1100 structure of the generative model, which may involve separately generating the ordinal aspects of 1101 spoken language from its content. Technically, this would involve an interaction between —or coupling 1102 of - separate ordinal and content factors (Dehaene et al., 2015; Friston and Buzsaki, 2016). In other 1103 words, we would have to replace the probability transition matrices $(\mathbf{B})$ above with high dimensional 1104 arrays, so that the probability transitions among the levels of one factor depend upon the level of 1105 another. Note that learning the factorial structure of natural language is the focus of much work: e.g., 1106 neural language modelling using recurrent neural networks (Bengio et al., 2003; Mikolov, 2010; 1107 Mikolov et al., 2013; Shang et al., 2015), or sequence-to-sequence modelling (Bahdanau et al., 2014; 1108 Ghazvininejad et al., 2018; Sutskever et al., 2014; Vinyals and Le, 2015).

1109 We have not considered language acquisition; e.g. via the learning of the $\mathbf{A}, \mathbf{B}$ and $\mathbf{D}$ parameters above 1110 (Al-Muhaideb and Menai, 2011; Bengio et al., 2009; Friston et al., 2016). In principle, by listening to 1111 an authoritative sequence of questions and answers, it should be possible to simulate language 1112 acquisition at various levels, via structure learning and Bayesian model reduction (Tervo et al., 2016). 1113 This has been pursued in the context of abstract rule learning (Friston et al., 2017b), but has not been 1114 applied in the present context. At this point, we get close to the problems addressed in computational 1115 linguistics, via the use of hierarchal Dirichlet processes (MacKay and Peto, 1995; Salakhutdinov et al., 1116 2013; Teh et al., 2006). In this setting, the key problem is to optimise the structure and hierarchal form 1117 of the model — and to know when to add an extra factor or level. It is possible that this structure learning 1118 problem may be usefully addressed with existing work on hierarchal Dirichlet process models and 1119 nonparametric Bayes (Goldwater, 2006); combined with the more top-down approach promoted in this 1120 work.

1121 Finally, our syntax factor is over-simplistic, encompassing only a handful of possibilities. This was 1122 sufficient for the simulations we presented, but will become important in applications of this kind of 1123 generative model. There is a substantial literature on cognitive models of syntax processing (for a recent 1124 review, see (Demberg and Keller, 2019)) and how listeners deal with semantic ambiguity (Altmann and 1125 Steedman, 1988; Bever, 1970; Gibson, 1998, 2000). Generally speaking, evidence from visual 
Modelling 'Twenty Questions'

1126 paradigms (Kamide et al., 2003) points to a predictive process, which is broadly consistent with active

1127 inference. It has also been proposed that syntax may itself be hierarchical (Van Schijndel et al., 2013).

1128 In summary, we have presented a generative model and inference scheme that is capable of simulating 1129 exchanges between synthetic subjects. This generative model is deep and hierarchical: inferences at the 1130 higher level inform words that are selected at the lower level—and these levels are nested, such that 1131 phrase-level inferences generate the words contained within the phrase, and then the lower level 'resets' 1132 for the next phrase. Our simulations of the "Twenty Questions" game show that agents can select the 1133 best questions - to ask of another - to reduce their uncertainty (in a Bayes optimal fashion) about the 1134 subject of conversation. We have also shown that, if the agent has precise beliefs about the nature of 1135 the scene, it can correctly answer another agent's questions. These types of exchanges demonstrate a 1136 convergence of beliefs, reflecting a successful linguistic exchange. We have also simulated situations 1137 where, if the agent has very imprecise beliefs, it will acknowledge its own uncertainty. If two agents 1138 both start with imprecise beliefs, then their generative models will converge, even though neither agent 1139 knows the veridical state of the scene. This type of setting could be considered as a folie à deux or an 1140 example of joint creative thinking. Finally, this formulation of communication makes predictions for 1141 neurophysiological responses, based on belief updating. It predicts violation responses, like P300 and 1142 N400 responses, when an answer is inconsistent with the agent's beliefs, and shows theta-gamma 1143 coupling as an emergent property of belief updating. Overall, we envisage that this model will be a 1144 useful starting point for simulating more complex linguistic exchanges - that include metacognition, or 1145 which simulate language acquisition.

\section{Software note}

1148 Although the generative model changes from application to application, the belief updates - and 1149 simulated neuronal responses - described in this paper are generic and can be implemented using 1150 standard routines (here spm_MDP_VB_X.m). These routines are available as Matlab code in the SPM 
Modelling 'Twenty Questions'

1151

1152

1153

1154 Acknowledgements

1155 The Wellcome Trust funded K.J.F. (Ref: 088130/Z/09/Z), E.H. (Ref: WT091681MA), and the

1156 Wellcome Centre for Human Neuroimaging (Ref: 203147/Z/16/Z), where this work was conducted.

1158

1159

\section{Disclosure statement}

1161 The authors have no disclosures or conflict of interest.

1163 Appendices

1164 Appendix 1 - Expected free energy: the variational free energy of a policy is a functional of an

1165 approximate posterior distribution over states $Q(s \mid \pi)$ given observed outcomes $o$, under a 1166 probabilistic generative model $P(o, s \mid \pi)$, given a policy $\pi$ :

$F=E_{Q(s \mid \pi)}[\ln Q(s \mid \pi)-\ln P(o, s \mid \pi)]$

$1167=\underbrace{D_{K L}[Q(s \mid \pi) \| P(s \mid \pi)]}_{\text {complexity }}-\underbrace{E_{Q(s \mid \pi)}[\ln P(o \mid s)]}_{\text {accuracy }}$

1168 The second equality expresses free energy as the difference between a Kullback-Leibler divergence

1169 (i.e., complexity) and the expected log likelihood (i.e., accuracy), given (observed) outcomes. 
Modelling 'Twenty Questions'

1170 In contrast, the expected free energy of a policy is an average over (unobserved) outcomes that

1171 determines the distribution over (future) states:

$$
\begin{aligned}
G & =E_{Q(o, s \mid \pi)}[\ln Q(s \mid \pi)-\ln P(o, s \mid \pi)] \\
& =E_{Q(o, s \mid \pi)}[\ln Q(s \mid \pi)-\ln P(s \mid o, \pi)-\ln P(o \mid \pi)]
\end{aligned}
$$

1173 Replacing the true predictive posterior with the approximate predictive posterior gives:

$$
\begin{aligned}
G & =E_{Q(o, s \mid \pi)}[\underbrace{\ln Q(s \mid \pi)-\ln Q(s \mid o, \pi)}_{\text {intrinsic value }}-\underbrace{\ln P(o \mid \pi)}_{\text {extrinsic value }}] \\
& =\underbrace{D_{K L}[Q(o \mid \pi) \| P(o \mid \pi)]}_{\text {risk }}+\underbrace{H[Q(o \mid s)]}_{\text {ambiguity }}
\end{aligned}
$$

1175 The first equality expresses expected free energy in terms of intrinsic and extrinsic value, while the

1176 second expression is an equivalent formulation in terms of the divergence between predicted and prior

1177 preferences over outcomes (i.e., risk) and expected uncertainty about outcomes, given their causes (i.e., 1178 ambiguity). By comparing equation A.3 with A.1, it can be seen that risk is expected complexity and ambiguity is expected inaccuracy, under a particular policy.

1180 Note that, for completeness and clarity, in the above equations (and the expressions in Table 1) we have conditioned prior preferences on policies. In practice, however, we assume that prior preferences do not 1182 depend upon policies.

1184 Appendix 2 - Belief updating: approximate Bayesian inference corresponds to minimising variational 1185 free energy, with respect to the sufficient statistics that constitute posterior beliefs. For generative 1186 models of discrete states, the free energy of hidden states and policies can be expressed as the (time1187 dependent) free energy under each policy plus the complexity incurred by posterior beliefs about (time1188 invariant) policies, where (with some simplifications): 
Modelling 'Twenty Questions'

1189

$$
\begin{aligned}
F[Q] & =D_{K L}[Q(s, \pi) \| P(s, \pi)]-\mathrm{E}_{Q}[\ln P(o \mid x)] \\
& =\sum_{\tau} \mathrm{E}_{Q}[F(\pi, \tau)]+D_{K L}[Q(\pi) \| P(\pi)] \\
& =\boldsymbol{\pi} \cdot(\ln \boldsymbol{\pi}+\mathbf{F}+\mathbf{G})
\end{aligned}
$$

1190 The free energy of hidden states under each policy is then given by:

$$
\begin{aligned}
\mathbf{F}_{\pi} & =\sum_{\tau} F(\pi, \tau) \\
F(\pi, \tau) & =\underbrace{E_{Q\left(s_{\tau-1} \mid \pi\right)}\left[D\left[Q\left(s_{\tau} \mid \pi\right) \| P\left(s_{\tau} \mid s_{\tau-1}, \pi\right)\right]\right]}_{\text {complexity }}-\underbrace{E\left[\ln P\left(o_{\tau} \mid s_{\tau}\right)\right]}_{\text {accuracy }} \\
& =\mathbf{s}_{\pi, \tau} \cdot\left(\ln \mathbf{s}_{\pi, \tau}-\ln \mathbf{B}_{\pi, \tau-1} \mathbf{s}_{\pi, \tau-1}-\ln \mathbf{A} \cdot o_{\tau}\right)
\end{aligned}
$$

1192 The expected free energy of a policy has a similar form, but the expectation is over hidden states and

1193 outcomes that have yet to be observed; namely, $Q\left(o_{\tau}, s_{\tau}\right)=P\left(o_{\tau} \mid s_{\tau}\right) Q\left(s_{\tau} \mid \pi\right)$.

$$
\begin{aligned}
\mathbf{G}_{\pi} & =\sum_{\tau} G(\pi, \tau) \\
G(\pi, \tau) & =-\underbrace{E_{Q}\left[\ln Q\left(s_{\tau} \mid o_{\tau}, \pi\right)-\ln Q\left(s_{\tau} \mid \pi\right)\right]}_{\text {intrinsic value }}-\underbrace{E_{Q}\left[\ln P\left(o_{\tau} \mid \pi\right)\right]}_{\text {extrinsic value }} \\
& =\underbrace{D_{K L}\left[Q\left(o_{\tau} \mid \pi\right) \| P\left(o_{\tau} \mid \pi\right)\right]}_{\text {risk }}+\underbrace{H\left[Q\left(o_{\tau} \mid s_{\tau}\right)\right]}_{\text {ambiguity }} \\
& =\mathbf{o}_{\pi, \tau} \cdot\left(\ln \mathbf{o}_{\pi, \tau}+\mathbf{C}_{\tau}\right)+\mathbf{H} \cdot \mathbf{s}_{\pi, \tau} \\
\mathbf{H} & =-\operatorname{diag}(\mathbf{A} \cdot \ln \mathbf{A}) \\
\mathbf{C}_{\tau} & =-\ln P\left(o_{\tau} \mid \pi\right)
\end{aligned}
$$

Please see Table 1 for a definition of the variables in these equations. 
Modelling 'Twenty Questions'

Table 1 - Expressions pertaining to models of discrete states: the shaded rows describe hidden states and

1197 auxiliary variables, while the remaining rows describe model parameters and functionals.

\begin{tabular}{|c|c|}
\hline Expression & Description \\
\hline $\begin{array}{l}o_{\tau} \in\{0,1\} \\
\mathbf{o}_{\tau}=\sum_{\pi} \boldsymbol{\pi}_{\pi} \cdot \mathbf{o}_{\pi, \tau} \in[0,1]\end{array}$ & Outcomes and their posterior expectations \\
\hline $\begin{array}{l}s_{\tau} \in\{0,1\} \\
\mathbf{s}_{\tau}=\sum_{\pi} \boldsymbol{\pi}_{\pi} \cdot \mathbf{s}_{\pi, \tau} \in[0,1]\end{array}$ & Hidden states and their posterior expectations \\
\hline $\mathbf{o}_{\pi, \tau}=\mathbf{A} \mathbf{s}_{\pi, \tau}$ & Expected outcome, under a particular policy \\
\hline $\begin{array}{l}\pi \in\{1, \ldots, K\} \\
\pi=\left(\pi_{1}, \ldots, \pi_{K}\right) \in[0,1]\end{array}$ & $\begin{array}{l}\text { Policies specifying state transitions and their } \\
\text { posterior expectations }\end{array}$ \\
\hline $\begin{array}{l}\mathbf{v}_{\pi, \tau}=\ln \mathbf{s}_{\pi, \tau}: \boldsymbol{\varepsilon}_{\pi, \tau}=\dot{\mathbf{v}}_{\pi, \tau} \\
\mathbf{s}_{\pi, \tau}=\sigma\left(\mathbf{v}_{\pi, \tau}\right)\end{array}$ & $\begin{array}{l}\text { Auxiliary variable representing depolarisation } \\
\text { and expected state, under a particular policy }\end{array}$ \\
\hline $\begin{aligned} \boldsymbol{\varepsilon}_{\pi, \tau} & =\ln \mathbf{B}_{\pi, \tau-1} \mathbf{s}_{\pi, \tau-1}+\ln \mathbf{B}_{\pi, \tau} \cdot \mathbf{s}_{\pi, \tau+1} \\
& +\ln \mathbf{A} \cdot o_{\tau}-\ln \mathbf{s}_{\pi, \tau}\end{aligned}$ & $\begin{array}{l}\text { Auxiliary variables representing state } \\
\text { prediction error }\end{array}$ \\
\hline A & $\begin{array}{l}\text { The likelihood of an outcome under each } \\
\text { hidden state }\end{array}$ \\
\hline $\mathbf{B}_{\pi, \tau}$ & $\begin{array}{l}\text { Time dependent probability transition matrices } \\
\text { specified by the policy }\end{array}$ \\
\hline
\end{tabular}




\begin{tabular}{|ll|}
\hline $\mathbf{C}_{\tau}=-\ln P\left(o_{\tau} \mid \pi\right)$ & Prior surprise about outcomes; i.e. prior cost or \\
& inverse preference \\
\hline $\mathbf{D}$ & (Empirical) Prior expectations about initial \\
& hidden states \\
$\mathbf{F}_{\pi}=\sum_{\tau} F(\pi, \tau)$ & Variational free energy for each policy \\
\hline $\mathbf{G}_{\pi}=\sum_{\tau} G(\pi, \tau)$ & Expected free energy for each policy \\
\hline $\mathbf{G}_{o}=G(o)$ & \\
\hline$\sum_{\pi} \exp \left(-\mathbf{G}_{\pi}\right)$ & Expected free energy for next outcome \\
\hline & \\
\hline
\end{tabular}

1198 


\section{References}

1202 Adams, R.A., Shipp, S., Friston, K.J., 2013. Predictions not commands: active inference in the motor system. Brain Struct Funct. 218, 611-643.

Al-Muhaideb, S., Menai, M.E., 2011. Evolutionary computation approaches to the Curriculum Sequencing problem. Natural Computing 10, 891-920.

1206 Allwood, J., Nivre, J., Ahlsén, E., 1992. On the semantics and pragmatics of linguistic 1207 feedback. Journal of semantics 9, 1-26.

1208 Altmann, G., Steedman, M., 1988. Interaction with context during human sentence processing. Arnal, L.H., Giraud, A.L., 2012. Cortical oscillations and sensory predictions. Trends Cogn Sci 16, 390-398.

1211 Arnone, D., Patel, A., Tan, G.M.-Y., 2006. The nosological significance of Folie à Deux: a 1212 review of the literature. Ann Gen Psychiatry 5, 11-11.

1213 Bahdanau, D., Cho, K., Bengio, Y., 2014. Neural machine translation by jointly learning to 1214 align and translate. arXiv preprint arXiv:1409.0473.

1215 Barlow, H., 1961. Possible principles underlying the transformations of sensory messages, in:

1216 Rosenblith, W. (Ed.), Sensory Communication. MIT Press, Cambridge, MA, pp. 217-234.

1217 Barlow, H.B., 1974. Inductive inference, coding, perception, and language. Perception 3, 1231218134.

1219 Barsalou, L.W., 2003. Situated simulation in the human conceptual system. Language and 1220 Cognitive Processes 18, 513-562.

1221 Bengio, Y., \#233, \#244, Louradour, m., Collobert, R., Weston, J., 2009. Curriculum learning, 1222 Proceedings of the 26th Annual International Conference on Machine Learning. ACM, 1223 Montreal, Quebec, Canada, pp. 41-48.

1224 Bengio, Y., Ducharme, R., Vincent, P., Jauvin, C., 2003. A neural probabilistic language model. Journal of machine learning research 3, 1137-1155. 
Modelling 'Twenty Questions'

1226 Bever, T.G., 1970. The cognitive basis for linguistic structures. Cognition and the development 1227 of language $279,1-61$.

1228 Bornkessel, I., Zysset, S., Friederici, A.D., von Cramon, D.Y., Schlesewsky, M., 2005. Who 1229 did what to whom? The neural basis of argument hierarchies during language comprehension. 1230 Neuroimage 26, 221-233.

1231 Braiman, C., Fridman, E.A., Conte, M.M., Voss, H.U., Reichenbach, C.S., Reichenbach, T., 1232 Schiff, N.D., 2018. Cortical Response to the Natural Speech Envelope Correlates with 1233 Neuroimaging Evidence of Cognition in Severe Brain Injury. Current biology : CB 28, 3833$1234 \quad 3839 . e 3833$.

1235 Brown, E.C., Brune, M., 2012. The role of prediction in social neuroscience. Front Hum 1236 Neurosci 6, 147.

1237 Catal, O., Nauta, J., Verbelen, T., Simoens, P., Dhoedt, B., 2019. Bayesian Policy Selection 1238 Using Active Inference. Workshop on "Structure \& Priors in Reinforcement Learning" at ICLR 12392019 : Proceedings. 2019.

1240 Chomsky, N., 2017. The language capacity: architecture and evolution. Psychon Bull Rev 24, $1241 \quad 200-203$.

1242 Clark, A., 2016. Surfing Uncertainty: Prediction, Action, and the Embodied Mind. Oxford 1243 University Press.

1244 Collins, A.G.E., Frank, M.J., 2013. Cognitive control over learning: Creating, clustering and 1245 generalizing task-set structure. Psychological review 120, 190-229.

1246 Collobert, R., Weston, J., Bottou, L., Karlen, M., Kavukcuoglu, K., Kuksa, P., 2011. Natural 1247 language processing (almost) from scratch. Journal of machine learning research 12, 249312482537. 
Modelling 'Twenty Questions'

1249 Constant, A., Ramstead, M.J.D., Veissiere, S.P.L., Friston, K., 2019. Regimes of Expectations:

1250 An Active Inference Model of Social Conformity and Human Decision Making. Front Psychol

$1251 \quad 10,679$.

1252 Coulson, S., King, J.W., Kutas, M., 1998. Expect the unexpected: Event-related brain response to morphosyntactic violations. Language and Cognitive Processes 13, 21-58.

Creanza, N., Feldman, M.W., 2014. Complexity in models of cultural niche construction with selection and homophily. Proceedings of the National Academy of Sciences of the United States of America 111, 10830-10837.

Das, A., Kottur, S., Gupta, K., Singh, A., Yadav, D., Moura, J.M., Parikh, D., Batra, D., 2017.

Visual dialog, Proceedings of the IEEE Conference on Computer Vision and Pattern Recognition, pp. 326-335.

Dauwels, J., 2007. On Variational Message Passing on Factor Graphs, 2007 IEEE International Symposium on Information Theory, pp. 2546-2550.

Davis, M.H., Johnsrude, I.S., 2003. Hierarchical processing in spoken language comprehension. Journal of Neuroscience 23, 3423-3431.

Dayan, P., Hinton, G.E., Neal, R.M., Zemel, R.S., 1995. The Helmholtz machine. Neural

1265 Comput 7, 889-904.

1266 de Lafuente, V., Jazayeri, M., Shadlen, M.N., 2015. Representation of accumulating evidence 1267 for a decision in two parietal areas. J Neurosci 35, 4306-4318.

1268 Dehaene, S., Meyniel, F., Wacongne, C., Wang, L., Pallier, C., 2015. The Neural 1269 Representation of Sequences: From Transition Probabilities to Algebraic Patterns and 1270 Linguistic Trees. Neuron 88, 2-19.

1271 Demberg, V., Keller, F., 2019. Cognitive Models of Syntax and Sentence Processing. Human 1272 Language: From Genes and Brains to Behavior. Cambridge, MA: MIT Press.

1273 Deneve, S., 2008. Bayesian spiking neurons I: inference. Neural Comput 20, 91-117. 
Modelling 'Twenty Questions'

1274

1275

1276

1277

1278

1279

1280

1281

1282

1283

1284

1285

1286

1287

1288

1289

1290

1291

1292

1293

1294

1295

1296

1297

Devlin, J., Chang, M.-W., Lee, K., Toutanova, K., 2018. Bert: Pre-training of deep bidirectional transformers for language understanding. arXiv preprint arXiv:1810.04805.

Donchin, E., Coles, M.G.H., 1988. Is the P300 component a manifestation of context updating? Behavioral and Brain Sciences 11, 357.

Dowty, D.R., Karttunen, L., Zwicky, A.M., 1985. Natural language parsing: Psychological, computational, and theoretical perspectives, in: Joshi, A.K. (Ed.), Tree adjoining grammars: How much context-sensitivity is required to provide reasonable structural descriptions? Cambridge Univ. Press, Cambridge, UK, pp. 206 - 250.

Edwards, E., Chang, E.F., 2013. Syllabic ( approximately 2-5 Hz) and fluctuation ( approximately 1-10 Hz) ranges in speech and auditory processing. Hearing research $305,113-$ 134.

Elman, J.L., 1990. Finding structure in time. Cognitive science 14, 179-211.

Ferro, M., Ognibene, D., Pezzulo, G., Pirrelli, V., 2010. Reading as active sensing: a computational model of gaze planning during word recognition. Frontiers in Neurorobotics 4, 1.

Fleming, S.M., Dolan, R.J., Frith, C.D., 2012. Metacognition: computation, biology and function. Philos Trans R Soc Lond B Biol Sci. 367, 1280-1286.

Foerster, J.N., Assael, Y.M., de Freitas, N., Whiteson, S., 2016. Learning to communicate to solve riddles with deep distributed recurrent q-networks. arXiv preprint arXiv:1602.02672.

Foerster, J.N., Song, F., Hughes, E., Burch, N., Dunning, I., Whiteson, S., Botvinick, M., Bowling, M., 2018. Bayesian action decoder for deep multi-agent reinforcement learning. arXiv preprint arXiv:1811.01458.

Friederici, A.D., 2011. The Brain Basis of Language Processing: From Structure to Function. Physiological Reviews 91, 1357-1392. 
Modelling 'Twenty Questions'

1298 Friston, K., 2010. The free-energy principle: a unified brain theory? Nat Rev Neurosci. 11, $1299 \quad 127-138$.

1300 Friston, K., 2013. Life as we know it. J R Soc Interface 10, 20130475.

1301 Friston, K., Buzsaki, G., 2016. The Functional Anatomy of Time: What and When in the Brain. 1302 Trends Cogn Sci.

1303 Friston, K., FitzGerald, T., Rigoli, F., Schwartenbeck, P., O'Doherty, J., Pezzulo, G., 2016.

1304 Active inference and learning. Neuroscience and biobehavioral reviews 68, 862-879.

1305 Friston, K., FitzGerald, T., Rigoli, F., Schwartenbeck, P., Pezzulo, G., 2017a. Active Inference: 1306 A Process Theory. Neural Comput 29, 1-49.

1307 Friston, K., Frith, C., 2015a. A Duet for one. Conscious Cogn 36, 390-405.

1308 Friston, K., Levin, M., Sengupta, B., Pezzulo, G., 2015. Knowing one's place: a free-energy 1309 approach to pattern regulation. J R Soc Interface 12.

1310 Friston, K., Mattout, J., Trujillo-Barreto, N., Ashburner, J., Penny, W., 2007. Variational free energy and the Laplace approximation. NeuroImage 34, 220-234.

1312 Friston, K., Penny, W., 2011. Post hoc Bayesian model selection. Neuroimage 56, 2089-2099.

1313 Friston, K., Schwartenbeck, P., FitzGerald, T., Moutoussis, M., Behrens, T., Dolan, R.J., 2014.

1314 The anatomy of choice: dopamine and decision-making. Philosophical transactions of the 1315 Royal Society of London. Series B, Biological sciences 369.

1316 Friston, K.J., Frith, C.D., 2015b. Active inference, communication and hermeneutics(). Cortex; 1317 a journal devoted to the study of the nervous system and behavior $68,129-143$.

1318 Friston, K.J., Lin, M., Frith, C.D., Pezzulo, G., Hobson, J.A., Ondobaka, S., 2017b. Active 1319 Inference, Curiosity and Insight. Neural Comput 29, 2633-2683.

1320 Friston, K.J., Parr, T., de Vries, B., 2017c. The graphical brain: Belief propagation and active 1321 inference. Network neuroscience (Cambridge, Mass.) 1, 381-414. 
Modelling 'Twenty Questions'

1322 Friston, K.J., Rosch, R., Parr, T., Price, C., Bowman, H., 2017d. Deep temporal models and 1323 active inference. Neuroscience and biobehavioral reviews 77, 388-402.

1324 Frith, C., Wentzer, T., 2013. Neural Hermeneutics, in: Kaldis, B. (Ed.), Encyclopedia of 1325 Philosophy and the Social Sciences. SAGE publications, Inc, Thousand Oaks.

1326 Garrod, S., Pickering, M.J., 2009. Joint Action, Interactive Alignment, and Dialog. Topics in 1327 Cognitive Science 1, 292-304.

1328 George, D., Hawkins, J., 2009. Towards a mathematical theory of cortical micro-circuits. PLoS 1329 Comput Biol 5, e1000532.

1330 Gershman, S.J., 2017. Predicting the Past, Remembering the Future. Curr Opin Behav Sci 17, $1331 \quad 7-13$.

1332 Ghazanfar, A.A., Takahashi, D.Y., 2014. The evolution of speech: vision, rhythm, cooperation. 1333 Trends Cogn Sci 18, 543-553.

1334 Ghazvininejad, M., Brockett, C., Chang, M.-W., Dolan, B., Gao, J., Yih, W.-t., Galley, M., 1335 2018. A knowledge-grounded neural conversation model, Thirty-Second AAAI Conference on 1336 Artificial Intelligence.

1337 Gibson, E., 1998. Linguistic complexity: Locality of syntactic dependencies. Cognition 68, 1133876

1339 Gibson, E., 2000. The dependency locality theory: A distance-based theory of linguistic 1340 complexity. Image, language, brain 2000, 95-126.

1341 Giraud, A.L., Poeppel, D., 2012. Cortical oscillations and speech processing: emerging 1342 computational principles and operations. Nat Neurosci 15, 511-517.

1343 Goldwater, S., 2006. Nonparametric Bayesian Models of Lexical Acquisition. Brown 1344 University.

1345 Gregory, R.L., 1980. Perceptions as hypotheses. Phil Trans R Soc Lond B. 290, 181-197. 
Modelling 'Twenty Questions'

1346 Hale, J., 2001. A probabilistic Earley parser as a psycholinguistic model, Proceedings of the 1347 second meeting of the North American Chapter of the Association for Computational 1348 Linguistics on Language technologies. Association for Computational Linguistics, pp. 1-8.

1349 Hassabis, D., Maguire, E.A., 2007. Deconstructing episodic memory with construction. Trends 1350 Cogn Sci 11, 299-306.

1351 Hauser, M.D., Chomsky, N., Fitch, W.T., 2002. The faculty of language: What is it, who has 1352 it, and how did it evolve? Science 298, 1569-1579.

1353 Hawkins, R.X., Stuhlmüller, A., Degen, J., Goodman, N.D., 2015. Why do you ask? Good 1354 questions provoke informative answers. Citeseer.

Helmholtz, H., 1878 (1971). The Facts of Perception, in: Middletown, R.K. (Ed.), The Selected Writings of Hermann von Helmholtz. Wesleyan University Press, Connecticut, p. 384. Hoeting, J.A., Madigan, D., Raftery, A.E., Volinsky, C.T., 1999. Bayesian Model Averaging: 1358 A Tutorial. Statistical Science 14, 382-401.

1359 Hohwy, J., 2016. The Self-Evidencing Brain. Noûs 50, 259-285.

1360 Hovsepyan, S., Olasagasti, I., Giraud, A.-L., 2018. Combining predictive coding with neural 1361 oscillations optimizes on-line speech processing. bioRxiv, 477588.

1362 Howard, R., 1966. Information Value Theory. IEEE Transactions on Systems, Science and 1363 Cybernetics SSC-2, 22-26.

1364 Hyafil, A., Cernak, M., 2015. Neuromorphic based oscillatory device for incremental syllable 1365 boundary detection. Idiap.

1366 Isomura, T., Parr, T., Friston, K., 2019. Bayesian Filtering with Multiple Internal Models: 1367 Toward a Theory of Social Intelligence. Neural Comput, 1-42.

1368 Itti, L., Baldi, P., 2009. Bayesian Surprise Attracts Human Attention. Vision Res. 49, 129513691306. 
Modelling 'Twenty Questions'

1370 Jaakkola, T., Jordan, M., 1998. Improving the Mean Field Approximation Via the Use of 1371 Mixture Distributions, in: Jordan, M. (Ed.), Learning in Graphical Models, vol. 89. Springer 1372 Netherlands, pp. 163-173.

1373 Kamide, Y., Scheepers, C., Altmann, G.T., 2003. Integration of syntactic and semantic 1374 information in predictive processing: Cross-linguistic evidence from German and English. 1375 Journal of psycholinguistic research 32, 37-55.

1376 Kayser, C., 2019. Evidence for the Rhythmic Perceptual Sampling of Auditory Scenes. Front 1377 Hum Neurosci 13, 249.

1378 Khani, F., Goodman, N.D., Liang, P., 2018. Planning, Inference and Pragmatics in Sequential 1379 Language Games. Transactions of the Association for Computational Linguistics 6, 543-555. 1380 Kiebel, S.J., Daunizeau, J., Friston, K.J., 2009. Perception and hierarchical dynamics. Front 1381 Neuroinform 3, 20.

1382 Kira, S., Yang, T., Shadlen, M.N., 2015. A neural implementation of Wald's sequential 1383 probability ratio test. Neuron $85,861-873$.

1384 Kschischang, F.R., Frey, B.J., Loeliger, H.A., 2001. Factor graphs and the sum-product 1385 algorithm. Ieee T Inform Theory 47, 498-519.

1386 Kuchling, F., Friston, K., Georgiev, G., Levin, M., 2019. Morphogenesis as Bayesian 1387 inference: A variational approach to pattern formation and control in complex biological 1388 systems. Phys Life Rev.

1389 Kutas, M., Hillyard, S.A., 1984. Brain potentials during reading reflect word expectancy and 1390 semantic association. Nature 307, 161.

1391 Leonard, M.K., Baud, M.O., Sjerps, M.J., Chang, E.F., 2016. Perceptual restoration of masked 1392 speech in human cortex. Nat Commun 7, 13619.

1393 Levy, R., 2008. Expectation-based syntactic comprehension. Cognition 106, 1126-1177. 
Modelling 'Twenty Questions'

1394 Li, J., Monroe, W., Ritter, A., Galley, M., Gao, J., Jurafsky, D., 2016. Deep reinforcement 1395 learning for dialogue generation. arXiv preprint arXiv:1606.01541.

1396 Lightfoot, D.W., 1979. Principles of diachronic syntax. Cambridge Univ. Press, Cambridge, 1397 UK, and New York.

1398 Lindley, D.V., 1956. On a Measure of the Information Provided by an Experiment. Ann. Math. 1399 Statist. 27, 986-1005.

1400 Linsker, R., 1990. Perceptual neural organization: some approaches based on network models 1401 and information theory. Annu Rev Neurosci. 13, 257-281.

1402 Liu, X., Duh, K., Gao, J., 2018. Stochastic answer networks for natural language inference. 1403 arXiv preprint arXiv:1804.07888.

Lizarazu, M., Lallier, M., Molinaro, N., 2019. Phase-amplitude coupling between theta and gamma oscillations adapts to speech rate. Ann N Y Acad Sci.

1406 MacKay, D.J.C., Peto, L.C.B., 1995. A hierarchical Dirichlet language model. Natural 1407 Language Engineering 1, 289-308.

1408 Maisto, D., Donnarumma, F., Pezzulo, G., 2015. Divide et impera: subgoaling reduces the complexity of probabilistic inference and problem solving. 12, 20141335.

1410 Mar, R.A., 2011. The Neural Bases of Social Cognition and Story Comprehension, in: Fiske, 1411 S.T., Schacter, D.L., Taylor, S.E. (Eds.), Annual Review of Psychology, Vol 62, vol. 62, pp. $1412 \quad 103-134$.

1413 Mathewson, K.W., Castro, P.S., Cherry, C., Foster, G., Bellemare, M.G., 2019. Shaping the 1414 Narrative Arc: An Information-Theoretic Approach to Collaborative Dialogue. arXiv preprint 1415 arXiv:1901.11528.

1416 Mattys, S.L., White, L., Melhorn, J.F., 2005. Integration of multiple speech segmentation cues: 1417 A hierarchical framework. Journal of Experimental Psychology-General 134, 477-500.

1418 Mikolov, T., 2010. Statistical language models based on neural networks. 
Modelling 'Twenty Questions'

1419 Mikolov, T., Yih, W.-t., Zweig, G., 2013. Linguistic regularities in continuous space word 1420 representations, Proceedings of the 2013 Conference of the North American Chapter of the 1421 Association for Computational Linguistics: Human Language Technologies, pp. 746-751.

1422 Mirza, M.B., Adams, R.A., Mathys, C.D., Friston, K.J., 2016. Scene Construction, Visual 1423 Foraging, and Active Inference. Frontiers in computational neuroscience 10, 56.

1424 Norris, D., McQueen, J.M., Cutler, A., 2016. Prediction, Bayesian inference and feedback in 1425 speech recognition. Language, cognition and neuroscience 31, 4-18.

1426 Ognibene, D., Baldassarre, G., 2014. Ecological Active Vision: Four Bio-Inspired Principles 1427 to Integrate Bottom-Up and Adaptive Top-Down Attention Tested With a Simple Camera-Arm 1428 Robot, IEEE Transactions onAutonomous Mental Development. IEEE, p. 99.

1429 Optican, L., Richmond, B.J., 1987. Temporal encoding of two-dimensional patterns by single 1430 units in primate inferior cortex. II Information theoretic analysis. J Neurophysiol. 57, 132-146. 1431 Page, M.P., Norris, D., 1998. The primacy model: a new model of immediate serial recall. 1432 Psychol Rev 105, 761-781.

1433 Parr, T., Friston, K.J., 2018. The Anatomy of Inference: Generative Models and Brain 1434 Structure. Frontiers in computational neuroscience 12.

1435 Parr, T., Markovic, D., Kiebel, S.J., Friston, K.J., 2019. Neuronal message passing using Mean1436 field, Bethe, and Marginal approximations. Scientific reports 9, 1889.

1437 Pefkou, M., Arnal, L.H., Fontolan, L., Giraud, A.L., 2017. theta-Band and beta-Band Neural 1438 Activity Reflects Independent Syllable Tracking and Comprehension of Time-Compressed 1439 Speech. J Neurosci 37, 7930-7938.

1440 Penn, D.C., Holyoak, K.J., Povinelli, D.J., 2008. Darwin's mistake: Explaining the 1441 discontinuity between human and nonhuman minds. Behavioral and Brain Sciences 31, 109-+. 
Modelling 'Twenty Questions'

1442 Pennington, J., Socher, R., Manning, C., 2014. Glove: Global vectors for word representation, 1443 Proceedings of the 2014 conference on empirical methods in natural language processing 1444 (EMNLP), pp. 1532-1543.

1445 Potts, C., 2012. Goal-driven answers in the Cards dialogue corpus.

1446 Pulvermuller, F., Lutzenberger, W., Birbaumer, N., 1995. Electrocortical distinction of 1447 vocabulary types. Electroencephalogr Clin Neurophysiol 94, 357-370.

1448 Purpura, K.P., Kalik, S.F., Schiff, N.D., 2003. Analysis of perisaccadic field potentials in the 1449 occipitotemporal pathway during active vision. J Neurophysiol 90, 3455-3478.

1450 Radford, A., Wu, J., Child, R., Luan, D., Amodei, D., Sutskever, I., 2019. Language models 1451 are unsupervised multitask learners.

1452 Rizzolatti, G., Craighero, L., 2004. The mirror-neuron system. Annual Review of Neuroscience $145327,169-192$.

1454 Rosenfeld, R., 2000. Two decades of statistical language modeling: Where do we go from here? 1455 Proceedings of the Ieee 88, 1270-1278.

1456 Roy, D., 2005. Semiotic schemas: A framework for grounding language in action and 1457 perception. Artificial Intelligence 167, 170-205.

1458 Salakhutdinov, R., Tenenbaum, J.B., Torralba, A., 2013. Learning with hierarchical-deep 1459 models. IEEE transactions on pattern analysis and machine intelligence 35, 1958-1971.

1460 Sallans, B., Hinton, G.E., 2004. Reinforcement Learning with Factored States and Actions. 1461 Journal of Machine Learning Research 5, 1063-1088.

1462 Schegloff, E.A., Sacks, H., 1973. Opening up closings. Semiotica 8, 289-327.

1463 Schmidhuber, J., 2006. Developmental robotics, optimal artificial curiosity, creativity, music, 1464 and the fine arts. Connection Science 18, 173-187. 
Modelling 'Twenty Questions'

1465 Schwartenbeck, P., FitzGerald, T.H., Mathys, C., Dolan, R., Friston, K., 2015. The 1466 Dopaminergic Midbrain Encodes the Expected Certainty about Desired Outcomes. Cereb 1467 Cortex 25, 3434-3445.

1468 Serban, I.V., Sordoni, A., Bengio, Y., Courville, A., Pineau, J., 2016. Building end-to-end 1469 dialogue systems using generative hierarchical neural network models, Thirtieth AAAI 1470 Conference on Artificial Intelligence.

1471 Shang, L., Lu, Z., Li, H., 2015. Neural responding machine for short-text conversation. arXiv 1472 preprint arXiv:1503.02364.

1473 Shea, N., Boldt, A., Bang, D., Yeung, N., Heyes, C., Frith, C.D., 2014. Supra-personal 1474 cognitive control and metacognition. Trends Cogn Sci 18, 186-193.

1475 Shipp, S., Adams, R.A., Friston, K.J., 2013. Reflections on agranular architecture: predictive 1476 coding in the motor cortex. Trends Neurosci. 36, 706-716.

1477 Singer, W., Sejnowski, T.J., Rakic, P., 2019. The neocortex.

1478 Sordoni, A., Bengio, Y., Vahabi, H., Lioma, C., Grue Simonsen, J., Nie, J.-Y., 2015. A 1479 hierarchical recurrent encoder-decoder for generative context-aware query suggestion, 1480 Proceedings of the 24th ACM International on Conference on Information and Knowledge 1481 Management. ACM, pp. 553-562.

1482 Specht, K., 2014. Neuronal basis of speech comprehension. Hearing research 307, 121-135. 1483 Steels, L., 2011. Modeling the cultural evolution of language. Physics of Life Reviews 8, 3391484356.

1485 Sutskever, I., Vinyals, O., Le, Q., 2014. Sequence to sequence learning with neural networks. 1486 Advances in NIPS.

1487 Teh, Y.W., Jordan, M.I., Beal, M.J., Blei, D.M., 2006. Hierarchical Dirichlet Processes. Journal 1488 of the American Statistical Association 101, 1566-1581. 
Modelling 'Twenty Questions'

1489 Tenenbaum, J.B., Kemp, C., Griffiths, T.L., Goodman, N.D., 2011. How to grow a mind: 1490 statistics, structure, and abstraction. Science 331, 1279-1285.

1491 Tervo, D.G., Tenenbaum, J.B., Gershman, S.J., 2016. Toward the neural implementation of 1492 structure learning. Curr Opin Neurobiol 37, 99-105.

1493 Van Petten, C., Coulson, S., Rubin, S., Plante, E., Parks, M., 1999. Time course of word 1494 identification and semantic integration in spoken language. Journal of Experimental 1495 Psychology: Learning, Memory, and Cognition 25, 394.

1496 Van Petten, C., Kutas, M., 1990. Interactions between sentence context and word 1497 frequencyinevent-related brainpotentials. Memory \& cognition 18, 380-393.

1498 Van Petten, C., Luka, B.J., 2012. Prediction during language comprehension: Benefits, costs, 1499 and ERP components. International Journal of Psychophysiology 83, 176-190.

1500 Van Schijndel, M., Exley, A., Schuler, W., 2013. A model of language processing as hierarchic 1501 sequential prediction. Topics in Cognitive Science 5, 522-540.

1502 Vaswani, A., Shazeer, N., Parmar, N., Uszkoreit, J., Jones, L., Gomez, A.N., Kaiser, Ł., 1503 Polosukhin, I., 2017. Attention is all you need, Advances in neural information processing 1504 systems, pp. 5998-6008.

1505 Vinyals, O., Le, Q., 2015. A neural conversational model. arXiv preprint arXiv:1506.05869.

1506 Wilson, B., Marslen-Wilson, W.D., Petkov, C.I., 2017. Conserved Sequence Processing in 1507 Primate Frontal Cortex. Trends Neurosci 40, 72-82.

1508 Wilson, M., Wilson, T., 2005. An oscillator model of the timing of turn-taking. Psychonomic 1509 Bulletin \& Review 12, 957-968.

1510 Winn, J., Bishop, C.M., 2005. Variational message passing. Journal of Machine Learning 1511 Research 6, 661-694. 
Modelling 'Twenty Questions'

1512 Ylinen, S., Huuskonen, M., Mikkola, K., Saure, E., Sinkkonen, T., Paavilainen, P., 2016.

1513 Predictive coding of phonological rules in auditory cortex: A mismatch negativity study. Brain 1514 and language $162,72-80$.

1515 Young, T., Hazarika, D., Poria, S., Cambria, E., 2018. Recent trends in deep learning based 1516 natural language processing. ieee Computational intelligenCe magazine 13, 55-75.

1517 Yufik, Y.M., 1998. Virtual Associative Networks: A framework for cognitive modeling, in: K. 1518 Pribram (ed.), Brain and Values, LEA, New Jersey, 109-177.

1519 Yufik, Y.M., 2019. The Understanding Capacity and Information Dynamics in the Human 1520 Brain. Entropy 21, 308, 1-38.

1521 Yuille, A., Kersten, D., 2006. Vision as Bayesian inference: analysis by synthesis? Trends 1522 Cogn Sci. 10, 301-308.

1523 Zhang, C., Butepage, J., Kjellstrom, H., Mandt, S., 2018. Advances in Variational Inference. 1524 IEEE transactions on pattern analysis and machine intelligence.

1525 Zhao, T., Zhao, R., Eskenazi, M., 2017. Learning discourse-level diversity for neural dialog 1526 models using conditional variational autoencoders. arXiv preprint arXiv:1703.10960. 